\title{
ON THE NUMBER OF LIMIT CYCLES OF PLANAR QUADRATIC VECTOR FIELDS WITH A PERTURBED CENTER
}

\author{
A. YU. FISHKIN
}

\begin{abstract}
We investigate the number of limit cycles of a planar quadratic vector field with a perturbed center-like singular point. An upper bound is obtained on the number of $\delta$-good limit cycles of such a vector field (Theorem 1 ). Here $\delta$ is a parameter characterizing the limit cycles: it shows how far those cycles are from the singular points of the vector field and from the infinite points. The bound also includes another parameter, $\kappa$, characterizing the vector field. More precisely, $\kappa$ gives an estimate on the distance from the vector field to the set consisting of quadratic vector fields with a line of singular points. Earlier, Ilyashenko and Llibre found a bound on the number of $\delta$-good limit cycles of those vector fields which are sufficiently far from the fields with a center-like singular point. Theorem 1 and that bound complement each other and yield a new bound on the number of $\delta$-good limit cycles of a quadratic vector field, regardless of its distance to the vector fields with a center-like singular point (Theorem 2).
\end{abstract}

\section{QUADRATIC VECTOR FIELDS AND THEIR LIMIT CYCLES}

A planar quadratic vector field is a vector field given by a system of differential equations of the form

$$
\left\{\begin{array}{l}
\dot{x}=P(x, y), \\
\dot{y}=Q(x, y),
\end{array}\right.
$$

where $P(x, y)$ and $Q(x, y)$ are quadratic polynomials in $(x, y) \in \mathbb{R}^{2}$. Recall that a limit cycle of a planar vector field is an isolated closed orbit homeomorphic to a circle. This paper deals with upper bounds on the number of limit cycles of quadratic vector fields.

Quadratic vector fields are rather well understood and have a number of remarkable properties:

1) any limit cycle of a quadratic vector field encircles exactly one singular point, which is a topological focus;

2) a quadratic vector field cannot have more than two focus-type singular points;

3) all limit cycles of a quadratic vector field, except possibly one, encircle the same focus.

Properties 1) and 2) are mentioned in Coppel's survey (see 11 and the references thereof). Property 3) is a recent result of Pingguang Zhang ([11]-[13]). Thus, to determine an upper bound on the number of limit cycles of a quadratic vector field, one can try to find a bound on the number of limit cycles that encircle a focus-type singular point.

2010 Mathematics Subject Classification. Primary 34C07; Secondary 37C10, 37C27.

Supported by the RFFI Grants 7-01-00017-a and 05-01-02801-CNRS_a). 
Kapteyn's normal form. Consider the vector field (1) with a focus-type singular point at the origin. We shall find a bound on the number of " $\delta$-good" limit cycles which encircle that point. To determine " $\delta$-good" limit cycles, we find Kapteyn's normal form of the vector field $([8,9])$. A linear change of phase variables and time results in the vector field

$$
\left\{\begin{array}{l}
\dot{x}=\lambda_{1} x-y-\lambda_{3} x^{2}+\left(2 \lambda_{2}+\lambda_{5}\right) x y+\lambda_{6} y^{2}, \\
\dot{y}=x+\lambda_{1} y+\lambda_{2} x^{2}+\left(2 \lambda_{3}+\lambda_{4}\right) x y-\lambda_{2} y^{2} .
\end{array}\right.
$$

Here the subscript $\lambda$ in the label (2ג) stands for a set of coefficients $\left(\lambda_{1}, \ldots, \lambda_{6}\right)$. Furthermore, using a homothety of the phase plane, we can normalize the coefficients of the quadratic monomials in the right-hand side as follows:

$$
\bar{\lambda}=\left(\lambda_{2}, \ldots, \lambda_{6}\right) \in \mathbb{S}^{4} \text {, i.e., }|\bar{\lambda}|=1 .
$$

Thus, $\lambda=\left(\lambda_{1}, \bar{\lambda}\right) \in \Lambda=\mathbb{R} \times \mathbb{S}^{4}$. System (2ג) with normalized coefficients $\lambda \in \Lambda$ is called Kapteyn's normal form of the quadratic vector field, and $\Lambda=\mathbb{R} \times \mathbb{S}^{4}$ is called the coefficient space of the normal form. Furthermore, when speaking of the vector field $(2 \lambda)$, we shall always mean the vector field given by that system of equations.

$\delta$-good limit cycles. Suppose $\delta>0$. Consider the disk of radius $1 / \delta$ centered at the origin of the phase plane $\mathbb{R}^{2}$. In that disk, we remove the $\delta$-neighborhoods of all singular points of the vector field $\left(22_{\lambda}\right.$ (including complex neighborhoods of complex singular points in $\left.\mathbb{C}^{2}\right)$, except for the origin. Denote the obtained set by $B(\lambda, \delta)$.

Definition 1. A limit cycle of the vector field $22_{\lambda}$ in Kapteyn's normal form is said to be $\delta$-good if it encircles the origin and is contained in $B(\lambda, \delta)$.

Parameters characterizing a vector field. In this paper, an important role is played by two quantities characterizing a quadratic vector field in Kapteyn's normal form. They are defined in terms of their distance from the coefficient set $\lambda$ of the vector field (2 2 ) to some special subsets $\Sigma$ and $\Xi$ in the coefficient space $\Lambda$. We shall now define those subsets.

Centers, the locus $\Sigma$ and vector fields close to centers. We want to find $\lambda \in \Lambda$ such that the vector field $(2 \lambda)$ has a center-like singularity at the origin. By a theorem of Dulac [2], conditions for a quadratic vector field in Kapteyn's normal form to have a center are given by the equations

$$
f_{1}(\lambda)=f_{2}(\lambda)=f_{3}(\lambda)=f_{4}(\lambda)=0,
$$

where the $f_{i}$ are polynomials in $\lambda$, given by

$$
f_{1}=\lambda_{1}, \quad f_{2}=\lambda_{5} p_{6}, \quad f_{3}=\lambda_{2} \lambda_{4} p_{6} p_{4}, \quad f_{4}=\lambda_{2} \lambda_{4} p_{6}^{2} p_{2},
$$

and the $p_{i}$ are of the form

$$
p_{6}=\lambda_{3}-\lambda_{6}, \quad p_{4}=\lambda_{4}+5\left(\lambda_{3}-\lambda_{6}\right), \quad p_{2}=\lambda_{6}\left(\lambda_{3}-2 \lambda_{6}\right)-\lambda_{2}^{2} .
$$

Definition 2. The ideal generated by the polynomials $f_{1}(\lambda), \ldots, f_{4}(\lambda)$ in the ring of polynomials in $\lambda$ is called the Dulac ideal for system $\left(22_{\lambda}\right)$ in Kapteyn's normal form, and $f_{1}(\lambda), \ldots, f_{4}(\lambda)$ are called the Dulac generators.

Remark 1. We defined the Dulac ideal for system $\left(22_{\lambda}\right)$ by describing its generators. Formally, the Dulac ideal for an elliptic family of vector fields is defined as the ideal (in the ring of holomorphic functions) generated by the coefficients of the series for the factor field (of a semiformal one-dimensional projection of the original family, [7, §12F]); generators of this ideal can easily be found by the method of undetermined coefficients from the definition. In the case of the family $\left(2_{\lambda}\right)$, those generators are of the form (4). 
Later on, we shall also define the Bautin ideal of the displacement function of the Poincaré map along the vector field $\left(2_{\lambda}\right)$, but the problem of finding canonical generators of that ideal appears to be computationally unfeasible. The coincidence of the Bautin ideal and the Dulac ideal ([7, Theorem 12.32]) is one of the key facts we are using in the proofs of some preliminary results.

Equations (3) define a subset $\Sigma \subset \Lambda$, called the locus of the Dulac ideal. Notice that $\Sigma$ is compact because $\Lambda$ is unbounded only along $\lambda_{1}$, but $\lambda_{1}=0$ on $\Sigma$.

Definition 3. Suppose $\sigma>0$. The vector field $(2 \lambda)$ is said to be $\sigma$-close to centers if $\operatorname{dist}(\lambda, \Sigma) \leq \sigma$. Otherwise it is said to be $\sigma$-distant from centers. The distance is defined via the Euclidean metric in $\mathbb{R}^{6}$.

Singular set $\Xi$ and vector fields which are far from singular fields. A general vector field $(2 \lambda)$ has isolated singular points. But in a degenerate (singular) case it may have a line of singular points. Let $\Xi$ be the set of parameters $\lambda \in \Lambda$ corresponding to that case. Singular quadratic vector fields have no limit cycles, but for parameters $\lambda$ close to $\Xi$, the problem of estimating the number of limit cycles becomes more difficult. For this reason, the distance from $\lambda$ to $\Xi$ plays an important role. It is convenient to replace the Euclidean distance and measure proximity, or the lack thereof, between $\lambda$ and $\Xi$ in the following special way.

Rewriting (2) in polar coordinates $x=r \cos \theta, y=r \sin \theta$, we have

$$
\dot{r}=r\left(\lambda_{1}+f_{\lambda}(\theta) r\right), \quad \dot{\theta}=1+g_{\lambda}(\theta) r,
$$

where

$$
\begin{aligned}
f_{\lambda}(\theta)= & -\lambda_{3} \cos ^{3} \theta+\left(3 \lambda_{2}+\lambda_{5}\right) \cos ^{2} \theta \sin \theta \\
& +\left(2 \lambda_{3}+\lambda_{4}+\lambda_{6}\right) \cos \theta \sin ^{2} \theta-\lambda_{2} \sin ^{3} \theta, \\
g_{\lambda}(\theta)= & \lambda_{2} \cos ^{3} \theta+\left(3 \lambda_{3}+\lambda_{4}\right) \cos ^{2} \theta \sin \theta \\
& -\left(3 \lambda_{2}+\lambda_{5}\right) \cos \theta \sin ^{2} \theta-\lambda_{6} \sin ^{3} \theta .
\end{aligned}
$$

Let $\Gamma=\{(r, \theta) \mid \dot{\theta}=0\}$ be an isocline of the vector field $\left(6_{\lambda}\right)$. Then

$$
\left.\dot{r}\right|_{\Gamma}=-\frac{H_{\lambda}}{g_{\lambda}^{2}}, \quad \text { where } H_{\lambda}=\lambda_{1} g_{\lambda}-f_{\lambda} .
$$

The trigonometric polynomial $H_{\lambda}(\theta)$ of degree 3 is identically zero if and only if $\Gamma$ consists entirely of singular points. This is exactly the case $\lambda \in \Xi$. Set

$$
\left\|H_{\lambda}\right\|_{2}=\sqrt{\int_{0}^{2 \pi} H_{\lambda}^{2}(\theta) d \theta} .
$$

Definition 4. Suppose $\kappa>0$. The vector field $\left(22_{\lambda}\right)$ is said to be $\kappa$-distant from singular fields if $\left\|H_{\lambda}\right\|_{2}>\kappa$.

\section{Statement of main Results. The Poincaré map}

After giving all necessary definitions in Section 1 we can state the main results of this paper.

Theorem 1. Suppose $0<\delta<1$ and $0<\kappa<1$. Set

$$
\sigma=\exp \left(-10^{73} \kappa^{-2} \delta^{-33}\right) \text {. }
$$

If $\lambda \in \Lambda$ and the vector field $22_{\lambda}$ is $\sigma$-close to centers and $\kappa$-distant from singular fields, then the number of its $\delta$-good limit cycles does not exceed

$$
\exp \left[\exp \left(10^{72} \kappa^{-2} \delta^{-33}\right)\right]=e^{\sigma^{-1 / 10}}
$$


The estimate on the number of limit cycles in Theorem 1 is too high, but this is the only estimate of that kind. Yu. S. Ilyashenko and J. Llibre ([5]) obtained a similar estimate for vector fields $\sigma$-distant from centers. We shall consider it in 7 It depends on $\sigma$ and grows without bound when $\sigma \rightarrow 0$. Using Theorem 1, which gives a uniform estimate for vector fields $\sigma$-close to centers for all sufficiently small $\sigma$, as well as the Ilyashenko-Llibre theorem, it is easy to prove the following result.

Theorem 2. Suppose $0<\delta<1$ and $0<\kappa<1$. If $\lambda \in \Lambda$ and the vector field (2, is $\kappa$-distant from singular fields, then the number of its $\delta$-good limit cycles does not exceed

$$
\exp \left[\exp \left(10^{77} \kappa^{-2} \delta^{-33}\right)\right]
$$

We shall prove Theorem 1 in Section 4, using preliminary results established later in Sections [5, 6] and in Subsection 8.3. Theorem 2] will be proved in Subsection 8.4.

The Poincaré map. To estimate the number of limit cycles of a vector field (2 $\left.2_{\lambda}\right)$ we use a standard method - the Poincaré map.

Consider the ray $\mathbb{R}^{+}=\{x>0\}$ and define the Poincaré map $P_{\lambda}(x): \mathbb{R}^{+} \rightarrow \mathbb{R}^{+}$ for the vector field $\left(22_{\lambda}\right)$. With each $x \in \mathbb{R}^{+}$the map $P_{\lambda}(x)$ associates the first return point of the orbit of the vector field, beginning at $x$, to the ray $\mathbb{R}^{+}$. It is clear that the Poincaré map is defined in a small half-neighborhood of zero because the vector field has a focus at the origin. By continuity, set $P_{\lambda}(0)=0$. Generally speaking, $P_{\lambda}(x)$ is not everywhere defined on $\mathbb{R}^{+}$. But if $P_{\lambda}(x)$ is defined on an interval $K \subset \mathbb{R}^{+}$, then, by the definition of the Poincaré map, the number of isolated zeros of its displacement function $D_{\lambda}(x)=P_{\lambda}(x)-x$ coincides with the number of limit cycles of the vector field (2, intersecting $K$.

For the proof of Theorem 1 we need an estimate on the number of isolated zeros of $D_{\lambda}(x)$ on some special interval of $\mathbb{R}^{+}$. In the next section we shall describe a method for obtaining such an estimate.

\section{An estimate on the number of Zeros of a Perturbation OF AN ANALYTIC FUNCTION}

In this section we shall provide an estimate on the number of isolated zeros in a given compact set in $\mathbb{C}$ for a small perturbation of an analytic function ([3]). The main difference between this theorem and the prior results is that it applies to analytic perturbations of identically zero functions. First, we need some definitions.

Ideals in rings of analytic functions. Let $\Delta_{\rho}(\xi) \subset \mathbb{C}^{n}$ denote the polydisk of polyradius $\rho=\left(\rho_{1}, \ldots, \rho_{n}\right)$ centered at $\xi \in \mathbb{C}^{n}$. We shall not indicate the dimension of a polydisk when it is clear from the context. For $U \subset \mathbb{C}^{n}$, we use $O(U)$ and $O(\bar{U})$ to denote the classes of functions holomorphic in $U$ and in a neighborhood $\bar{U}$, respectively. Let $O^{n}(\xi)$ be the ring of germs of analytic functions of $n$ variables at $\xi$. Ideals in $O\left(\overline{\Delta_{\rho}(\xi)}\right)$ will be denoted by $I_{\rho}(\xi)$, and in $O^{n}(\xi)$ by $I(\xi)$. For a function $f$ holomorphic in a neighborhood of $\xi$, we write $f \in I(\xi)$ if its germ at $\xi$ belongs to $I(\xi)$. The ideal generated by the functions $\left\{f_{0}, \ldots, f_{d}\right\} \subset O\left(\overline{\Delta_{\rho}(\xi)}\right)$ (or by their germs at $\xi$ ) will be denoted by $\left(f_{0}, \ldots, f_{d}\right)_{\rho}(\xi)$ (respectively, by $\left.\left(f_{0}, \ldots, f_{d}\right)(\xi)\right)$. For $f \in O\left(\overline{\Delta_{\rho}(\xi)}\right)$ we set

$$
\|f\|_{\rho, \xi}=\max _{t \in \overline{\Delta_{\rho}(\xi)}}|f(t)| \text {. }
$$

Definition 5 (The Bautin ideal, [10, 7]). Consider a function $f(x, \lambda)$ of several complex variables holomorphic in a neighborhood of $(0, \xi) \in \mathbb{C} \times \mathbb{C}^{n}$. The variable $x \in \mathbb{C}$ is said 
to be principal, whereas the remaining $n$ variables $\lambda=\left(\lambda_{1}, \ldots, \lambda_{n}\right) \in \mathbb{C}^{n}$ are parameters. Suppose

$$
f(x, \lambda)=\sum_{k \geq 0} f_{k}(\lambda) x^{k}
$$

is the Taylor series expansion in $x$ of $f(x, \lambda)$ at the origin. The ideal $I=I(\xi)=$ $\left(f_{0}, \ldots, f_{k}, \ldots\right)(\xi)$, generated by all the coefficients $\left\{f_{k}\right\}$ at $\xi$, is called the Bautin ideal of the germ of $f$ at $x=0$ for $\lambda=\xi$.

Since the ring $O^{n}(\xi)$ is Noetherian ([4, Theorem 7]), any strictly increasing sequence of ideals in it is finite. This motivates the following definition.

Definition 6. The Bautin index of series (10) is the smallest $d \geq 0$ such that $I=$ $\left(f_{0}, \ldots, f_{d}\right)(\xi)$. Such $f_{0}, \ldots, f_{d}$ are called canonical generators of the Bautin ideal.

Ideals in the ring of holomorphic functions on a polydisk (in particular, Bautin ideals) are related to an important concept of growth constant. That quantity depends on the chosen system of generators for the ideal and bounds the moduli of the coefficients in the decomposition of elements of the ideal as linear combinations of the chosen generators. Here is a rigorous definition.

Definition 7. Suppose $\left\{f_{0}, \ldots, f_{n}\right\} \subset O\left(\overline{\Delta_{\rho}(\xi)}\right)$ and $I_{\rho}(\xi)=\left(f_{0}, \ldots, f_{n}\right)_{\rho}(\xi)$. We say that $C>0$ is a growth constant for decompositions of elements of $I_{\rho}(\xi)$ as linear combinations of $f_{0}, \ldots, f_{n}$ (or, simply, a growth constant for $I_{\rho}(\xi)$ and generators $f_{0}, \ldots, f_{n}$ ) if for any $f \in I_{\rho}(\xi)$ there is a decomposition

$$
f=\sum_{i=0}^{n} a_{i} f_{i}
$$

with $a_{i} \in O\left(\overline{\Delta_{\rho}(\xi)}\right)$ such that for any $i$,

$$
\left\|a_{i}\right\|_{\rho, \xi} \leq C\|f\|_{\rho, \xi} .
$$

Suppose $\left\{f_{0}, \ldots, f_{n}\right\} \subset O\left(\overline{\Delta_{\rho}(\xi)}\right), I_{\rho}(\xi)=\left(f_{0}, \ldots, f_{n}\right)_{\rho}(\xi)$ and $\Sigma(\rho, \xi)=\left\{\lambda \in \overline{\Delta_{\rho}(\xi)} \mid\right.$ $\left.f_{0}(\lambda)=\cdots=f_{n}(\lambda)=0\right\}$ is the locus of $I_{\rho}(\xi)$ inside the polydisk $\Delta_{\rho}(\xi)$. We want to define a notion of a suitable polydisk for $I_{\rho}(\xi)$. Set

$$
J_{\rho}(\xi)=\left\{f \in O\left(\overline{\Delta_{\rho}(\xi)}\right) \mid f \in I(\eta)=\left(f_{0}, \ldots, f_{n}\right)(\eta) \forall \eta \in \Sigma(\rho, \xi)\right\} .
$$

It is clear that $I_{\rho}(\xi) \subseteq J_{\rho}(\xi)$. The author does not know if the reverse inclusion holds in general.

Definition 8. We say that the polydisk $\Delta_{\rho}(\xi)$ is suitable for $I_{\rho}(\xi)$ if $J_{\rho}(\xi)=I_{\rho}(\xi)$.

It is easy to see that the property of the polydisk to be suitable does not depend on the choice of generators of the ideal.

Remark 2. We do not claim that a growth constant exists for an arbitrary polydisk or even for any suitable polydisk. The existence of a suitable polydisk and the existence of a growth constant for some choice of a suitable polydisk is proved in [4, Theorem 7]. Further arguments related to the growth constant make sense only when such a constant exists.

We need a few more definitions. The intrinsic diameter of a linearly connected compact set is the maximum of all shortest paths in this set between pairs of points. We write dist $(V, W)$ to denote the distance between two sets $V, W \subset \mathbb{C}$, understood as

$$
\operatorname{dist}(V, W)=\inf _{\substack{v \in V \\ w \in W}}|v-w| .
$$


An estimate on the number of zeros of a perturbation of an analytic function. The theorem below is proved in [3. It generalizes the Growth and Zeros Theorem in 6] and plays a key role in the proof of Theorem 1.

Theorem 3. Consider a linearly connected compactum $K \subset \mathbb{C}$, containing the disk $\overline{\Delta_{r}(0)}$ of radius $r \leq 1$, and:

- a simply connected neighborhood $U$ of $K$ with a piecewise smooth boundary,

- polyradius $R$ and a function $f(x, \lambda)$ holomorphic in $\overline{U \times \Delta_{R}(\xi)}$ with a modulus bounded there by a constant $M>0$,

- the Taylor series $f(x, \lambda)=\sum_{k>0} f_{k}(\lambda) x^{k}$ about $x=0$ with $\lambda \in \Delta_{R}(\xi)$, the corresponding Bautin ideal $I=I(\xi)$, and its index $d$.

There exists a polydisk $\Delta_{\rho}(\xi) \subset \Delta_{R}(\xi)$ such that for any $\lambda \in \overline{\Delta_{\rho}(\xi)}$ the number $N(\lambda)$ of isolated zeros of $f(\cdot, \lambda)$ in $K$ is bounded above by a constant depending only on the first Taylor coefficients $f_{0}, \ldots, f_{d}$ of series (10), on the quantity $M$, and on the geometry of $K$ and $U$. More precisely, let $D$ be the intrinsic diameter of $K, \varepsilon=\operatorname{dist}(K, \partial U)$, and $C(\rho, \xi)$ a growth constant for $I_{\rho}(\xi)=\left(f_{0}, \ldots, f_{d}\right)_{\rho}(\xi)$. Then

$$
N(\lambda) \leq \exp \left(\frac{2 D}{\varepsilon}\right) \ln \left(\frac{(d+1) C(\rho, \xi) M}{r^{d}}\right) .
$$

Moreover, (11) holds for any polydisk $\Delta_{\rho}(\xi) \subset \Delta_{R}(\xi)$, which is suitable for the ideal $I_{\rho}(\xi)$, with growth constant $C(\rho, \xi)$.

Remark 3. The last assertion of Theorem [3 on the number of zeros in an arbitrary suitable polydisk is not stated in [3, but the proof given there also works in this case.

Theorem 3 and an estimate on the number of zeros of $D_{\lambda}(x)$. Recall that $\Lambda$ is the coefficient space of Kapteyn's normal form $(2 \lambda)$ and $\Sigma \subset \Lambda$ is the subset corresponding to quadratic vector fields with center-like singularity at the origin. Clearly, when $\xi \in \Sigma$, the function $D_{\xi}(x)$ is identically zero for small $x>0$. Reformulating Theorem 3 for $f(x, \lambda)=D_{\lambda}(x)$ yields the following result.

Theorem 4. Consider $\delta \in(0,1)$ and an interval $K \subset \mathbb{R}^{+}$with the left endpoint at zero and whose length is at most $\frac{1}{\delta}$. Suppose that $D_{\lambda}(x)$ has an analytic continuation to a neighborhood of $K$ with respect to $x$ and a neighborhood of $\xi \in \Sigma$ with respect to $\lambda$. Suppose we can find

- an $\varepsilon$-neighborhood $U_{\varepsilon}(K)$ of $K$ in $\mathbb{C}$ with $\varepsilon \in(0,1)$ and

- a suitable polydisk $\Delta_{\rho}(\xi)$ for the Bautin ideal of $D_{\lambda}(x)$ at $x=0$ for $\lambda=\xi$

such that $D_{\lambda}(x)$ is holomorphic in $\left\{(x, \lambda) \in U_{\varepsilon}(K) \times \Delta_{\rho}(\xi)\right\}$ with modulus bounded there by a constant $M$. Suppose $C(\rho, \xi)$ is a growth constant for the above Bautin ideal and its canonical generators in the polydisk $\Delta_{\rho}(\xi)$. Then the number $N(\lambda)$ of zeros of $D_{\lambda}(x)$ in the interval $K$ with $\lambda \in \Delta_{\rho}(\xi)$ has the following estimate:

$$
N(\lambda) \leq \exp \left(1+\frac{2}{\delta \varepsilon}\right) \ln \left(\frac{2^{10} M C(\rho, \xi)}{\varepsilon^{7}}\right) .
$$

Proof. The Bautin ideal of $D_{\lambda}(x)$ at $x=0$ for $\lambda=\xi$ is well understood ([14, 7]). It is of index 7. Set $r=\frac{\varepsilon}{2}$ and $K^{\prime}=K \cup \Delta_{r}(0)$. We have the desired assertion by applying Theorem 3 to the function $f(x, \lambda)=D_{\lambda}(x)$, the compactum $K^{\prime}$, the domain $U_{\varepsilon}(K)$ and taking into account that $\operatorname{dist}\left(K^{\prime}, \partial U_{\varepsilon}(K)\right)=\frac{\varepsilon}{2}$ and that the intrinsic diameter of $K^{\prime}$ is at most $\frac{1}{\delta}+\frac{\varepsilon}{2}$. 


\section{Proof of Theorem 1}

Henceforth, unless stated otherwise, we shall use the following notation:

- $\Lambda, \Sigma, P_{\lambda}(x), D_{\lambda}(x)$ are defined as in Sections 1 and 2

- $I(\Sigma)$ (respectively, $I_{\rho}(\xi)$ ) is the Bautin ideal of $D_{\lambda}(x)$ at $x=0$ in the ring of germs of analytic functions on $\Sigma$ (respectively, in the $\operatorname{ring} O\left(\overline{\Delta_{\rho}(\xi)}\right)$ );

- $0<\delta<1$ and $0<\kappa<1$ are given values of the parameters from Theorem 1

- the auxiliary parameters $\beta, \gamma, \varepsilon$ are defined as follows:

$$
\begin{aligned}
\beta & =\frac{\delta^{15} \kappa}{10^{34}}, \\
\gamma & =\frac{\beta^{3} \delta^{6}}{25 \cdot 10^{5}} \exp \left(-\frac{2 \pi \cdot 10^{3}}{\beta^{2} \delta^{3}}\right) \\
\varepsilon & =\frac{\gamma \cdot 10^{4}}{\delta^{5} \beta^{2}}
\end{aligned}
$$

- $\omega=(\gamma, \gamma, \gamma, \gamma, \gamma, \gamma)$ is the polyradius with values $\gamma$;

- $\sigma=\exp \left(-10^{73} \kappa^{-2} \delta^{-33}\right)$;

- $\tilde{\lambda}$ is a fixed point of $\Lambda$ such that the vector field $(2 \tilde{\lambda})$ is $\kappa$-distant from singular fields, $\sigma$-close to centers, and has at least one $\delta$-good limit cycle (otherwise Theorem 1 is trivially true for $\tilde{\lambda}$ ).

We shall prove Theorem 1 with $\lambda=\tilde{\lambda}$ using Theorem 4, In this section we shall go through all the preparatory steps. First, we find an interval $K$ intersecting all $\delta$-good limit cycles of the vector field $(2 \tilde{\lambda})$. Next, we show, for $\varepsilon$ and $\omega$ defined above, that $D_{\lambda}(x)$ has an analytic continuation to $U_{\varepsilon}(K) \times \Delta_{\omega}(\tilde{\lambda})$ and has modulus bounded there by $M=\frac{2}{\delta}$. After that, for an arbitrary point $\xi \in \Sigma$ at a distance of at most $\sigma$ from $\tilde{\lambda}$, we construct a suitable for $I_{\rho}(\xi)$ polydisk $\Delta_{\rho}(\xi)$ and give an estimate for a growth function $C(\rho, \xi)$ for canonical generators of that ideal. We can choose $\rho$ such that

$$
\tilde{\lambda} \in \Delta_{\rho}(\xi) \subset \Delta_{\omega}(\tilde{\lambda}) .
$$

This implies that $D_{\lambda}(x)$ has an analytic continuation to $U_{\varepsilon}(K) \times \Delta_{\rho}(\xi)$. Applying Theorem 4 with the chosen $K, U_{\varepsilon}(K)$, and $\Delta_{\rho}(\xi)$ we shall have an estimate on the number of zeros of $D_{\lambda}(x)$ in $K$ for $\lambda \in \Delta_{\rho}(\xi)$, and, in particular, for $\lambda=\tilde{\lambda}$. By the definition of $K$, this is an upper bound for the number of $\delta$-good limit cycles of the vector field $(2 \tilde{\lambda})$.

Choosing interval K. Suppose $a(\tilde{\lambda})$ is the $x$-coordinate of the point of intersection of the outmost $\delta$-good limit cycle of the vector field $(2 \tilde{\lambda})$ (we denote this cycle by $L$ ) and the positive semi-axes $O x$. The value $a(\tilde{\lambda})$ is defined because we assume that the set of $\delta$-good limit cycles of this vector field is not empty. Set $K=[0, a(\tilde{\lambda})]$. By definition, $K$ intersects all $\delta$-good limit cycles of the vector field $\left(2_{\tilde{\lambda}}\right)$. The interval $K$ has the following property.

Lemma 1. The map $P_{\tilde{\lambda}}(x)$ is defined on the entire $K$.

Proof. Inside the domain bounded by the cycle $L$, there are no singular points of the vector field $\left(2_{\tilde{\lambda}}\right)$, except the origin. By the Poincaré-Bendixson theorem, the omega-limit set of any point $x$ in $K$ is either the origin (i.e., a focus) or a limit cycle encircling the origin. In both cases the orbit of $x$ intersects $O x$ infinitely many times. Therefore, there is the first point of intersection of this orbit and $O x$, and thus $P_{\tilde{\lambda}}(x)$ is defined on the entire $K$. 
Next we state two important lemmas that would allow us to use Theorem 4 and find an estimate on the number of zeros of $D_{\tilde{\lambda}}(x)$ in $K$.

Lemma 2. The function $D_{\tilde{\lambda}}(x)$ has an analytic continuation to $U_{\varepsilon}(K) \times \Delta_{\omega}(\tilde{\lambda})$ with modulus at most $\frac{2}{\delta}$ in that domain.

Lemma 3. Suppose $\xi \in \Sigma$ and $0<R<10^{-7}$. There is a polyradius $\rho=\left(\rho_{1}, \ldots, \rho_{6}\right)$ such that

$$
R<\rho_{i}<1000 \sqrt{R}
$$

and:

1) the polydisk $\Delta_{\rho}(\xi)$ is suitable for $I_{\rho}(\xi)$,

2) the growth constant for $I_{\rho}(\xi)$ and its canonical generators is at most $\frac{10^{56}}{R^{7}}$.

We shall prove Lemma 2 in Section 5 and Lemma 3 in Section 6 ,

Now we have all the necessary tools to finish the proof of Theorem [1. By assumption,

$$
\operatorname{dist}(\tilde{\lambda}, \Sigma) \leq \sigma .
$$

Suppose $\xi \in \Sigma$ is at a distance of at most $\sigma$ from $\tilde{\lambda}$. By Lemma 3, there is a suitable polydisk $\Delta_{\rho}(\xi)$ such that

$$
\sigma \leq \rho_{i} \leq 1000 \sqrt{\sigma}
$$

and the growth constant for canonical generators of the Bautin ideal $I_{\rho}(\xi)$ is at most $\frac{10^{56}}{\sigma^{7}}$. Furthermore, substituting in (14) the value of $\beta$ from (13), we have

$$
\gamma=\frac{\delta^{51} \kappa^{3}}{25 \cdot 10^{107}} \exp \left(-2 \pi \cdot 10^{71} \kappa^{-2} \delta^{-33}\right)
$$

It is easy to see that, replacing $2 \pi$ by 8 in the exponent, we have

$$
\gamma>\exp \left(-8 \cdot 10^{71} \kappa^{-2} \delta^{-33}\right)
$$

Furthermore, by the choice of $\sigma$,

$$
1000 \sqrt{\sigma}<\gamma
$$

Therefore,

$$
\tilde{\lambda} \in \Delta_{\rho}(\xi) \subset \Delta_{\omega}(\tilde{\lambda}),
$$

and, by Lemma 2 the map $D_{\lambda}$ has an analytic continuation to $U_{\varepsilon}(K) \times \Delta_{\rho}(\xi)$. Similar to (16), one can show that

$$
\varepsilon=\frac{\gamma \cdot 10^{4}}{\delta^{5} \beta^{2}}>\exp \left(-9 \cdot 10^{71} \kappa^{-2} \delta^{-33}\right) .
$$

Applying Theorem 4 and rewriting (12), we have

$$
\begin{aligned}
N(\lambda) & \leq \exp \left(1+\frac{2}{\delta \varepsilon}\right) \ln \left(\frac{2^{11} \cdot \delta^{-1} \cdot 10^{56} \cdot \sigma^{-7}}{\varepsilon^{7}}\right) \\
& \leq e \cdot \exp \left[2 \delta^{-1} \exp \left(9 \cdot 10^{71} \kappa^{-2} \delta^{-33}\right)\right] \ln \left(\frac{2^{11} \cdot \delta^{-1} \cdot 10^{56} \cdot \sigma^{-7}}{\varepsilon^{7}}\right) \\
& \leq \exp \left[\exp \left(10^{72} \kappa^{-2} \delta^{-33}\right)\right] .
\end{aligned}
$$

Thus, we have obtained estimate (9) for the number of isolated zeros of $D_{\lambda}(x)$ in $K$ with $\lambda \in \Delta_{\rho}(\xi)$. For $\lambda=\tilde{\lambda} \in \Delta_{\rho}(\xi)$, by the definition of $K$, we found an estimate on the number of $\delta$-good limit cycles of the vector field $(2 \tilde{\lambda})$. Thus, with the aid of Lemmas 2 and 3, we have proved Theorem 1. 


\section{Proof of Lemma 2}

To prove Lemma 2 we need some preliminary results.

Proposition 1. If $\lambda \in \Lambda$ and the vector field [2] has $\delta$-good limit cycles, then $\left|\lambda_{1}\right| \leq \frac{10}{\delta}$.

Proof. When $\lambda \in \Lambda$ and $i \neq 1$ we have $\left|\lambda_{i}\right| \leq 1$. It now follows from (77) that $\left|f_{\lambda}\right| \leq 10$. If $\left|\lambda_{1}\right|>\frac{10}{\delta}$, then equations $\left(6_{\lambda}\right)$ show that $|\dot{r}|>0$ for $r \leq \frac{1}{\delta}$. Therefore, there are no limit cycles inside the circle of radius $\frac{1}{\delta}$. Hence there are no $\delta$-good limit cycles.

Consider the set of coefficients $\tilde{\lambda}$ and the interval $K$ chosen in 4 Our second preliminary result provides a lower estimate on the "gap" between $\delta$-good limit cycles of the vector field $\left(2_{\tilde{\lambda}}\right)$ and its isocline $\Gamma=\{\dot{\theta}=0\}$. In estimating this gap the main role is played by the $\kappa$-separation between the vector field $(2 \tilde{\lambda})$ and the singular fields. It follows from $\left(6_{\tilde{\lambda}}\right)$ that the isocline $\Gamma$ is given by the equation

$$
r=-\frac{1}{g_{\tilde{\lambda}}(\theta)} \text {. }
$$

Define a domain

$$
\begin{aligned}
\Omega_{\beta}= & \left\{(\theta, r) \mid 0<r<-\frac{1}{g_{\tilde{\lambda}}(\theta)}-\beta \text { if } g_{\tilde{\lambda}}(\theta)<0\right\} \\
& \cup\left\{(\theta, r) \mid 0<r<+\infty \text { if } g_{\tilde{\lambda}}(\theta) \geq 0\right\},
\end{aligned}
$$

where $\beta$ is given by (13). As (77) shows, $\left|g_{\tilde{\lambda}}(\theta)\right| \leq 10$ and therefore, if $g_{\tilde{\lambda}}(\theta)<0$,

$$
-\frac{1}{g_{\tilde{\lambda}}(\theta)}-\beta \geq 0.1-\beta>0
$$

which implies that $\Omega_{\beta}$ is indeed a domain.

Proposition 2. All $\delta$-good limit cycles of the vector field $\left(2_{\tilde{\lambda}}\right)$ lie in $\Omega_{\beta}$.

In Subsection 8.3 we shall state a similar result proved in [5]. Based on that, we shall then easily prove Proposition 2 ,

Now we prove Lemma 2. By Lemma 1, the function $D_{\tilde{\lambda}}(x)$ is defined for $x \in K$. To find an analytic continuation of $D_{\tilde{\lambda}}(x)$ in $x$ and in $\lambda$, consider system $\left(6_{\lambda}\right)$ in polar coordinates as well as the corresponding nonautonomous equation obtained by eliminating time. If we replace the polar radius by a complex variable $z$ but still keep $\theta \in[0,2 \pi]$ real, then the equation becomes

$$
\frac{d z}{d \theta}=z \frac{\lambda_{1}+z f_{\lambda}(\theta)}{1+z g_{\lambda}(\theta)}, \quad z \in \mathbb{C}, \quad \theta \in \mathbb{S}^{1} .
$$

For brevity, we denote the right-hand side of this equation by $F_{\lambda}(z, \theta)$. Now we allow the components of the vector parameter $\lambda$ to take on complex values. Suppose that $z(\lambda, \theta, x)$ is the solution of (17) with initial value $z(\lambda, 0, x)=x \in \mathbb{C}$. By the analytic dependence of solutions on initial conditions and parameters, the function $P_{\lambda}(x)=z(\lambda, 2 \pi, x)$ is, wherever it is defined, an analytic continuation in $x$ and in $\lambda$ of the Poincaré map.

Let $D$ be the domain in $\mathbb{R}^{+} \times \mathbb{S}^{1}$ bounded by the outmost $\delta$-good limit cycle $L$ of the vector field $(2 \tilde{\lambda})$ which passes through the right endpoint of $K$ :

$$
L=\{z(\tilde{\lambda}, \theta, a(\tilde{\lambda})) \mid \theta \in[0,2 \pi]\} .
$$

It follows from Proposition 2 that $D \subset \Omega_{\beta}$. Consider the $\frac{\beta \delta}{25}$-neighborhood $D^{\prime}$ of $D$ in $\mathbb{C} \times \mathbb{S}^{1}$ (for each $\theta$ we take the $\frac{\beta \delta}{25}$-neighborhood of the interval $[0, z(\tilde{\lambda}, \theta, a(\tilde{\lambda}))]$ in $\left.\mathbb{C}\right)$. We shall show that, for $\lambda \in \Delta_{\omega}(\tilde{\lambda})$ and $x \in U_{\varepsilon}(K)$, the solution $z(\lambda, \theta, x)$ of (17) is defined when $\theta \in[0,2 \pi]$ and is contained in $D^{\prime}$. By analytic dependence of solutions on 
initial conditions and parameters, this implies that $P_{\lambda}(x)=z(\lambda, 2 \pi, x)$ is analytic in $x$ and $\lambda$ in $U_{\varepsilon}(K) \times \Delta_{\omega}(\tilde{\lambda})$. Moreover, since $P_{\lambda}(x) \in U_{\beta \delta / 25}(K)$ when $x \in U_{\varepsilon}(K)$, and the length of $K$ is at most $\frac{1}{\delta}$, we have, using a rough estimate,

$$
\left|D_{\lambda}(x)\right|=\left|P_{\lambda}(x)-x\right| \leq \varepsilon+\frac{\beta \delta}{25}+\frac{1}{\delta}<\frac{2}{\delta} .
$$

This would prove Lemma 2 .

To show that for $\lambda \in \Delta_{\omega}(\tilde{\lambda})$ and $x \in U_{\varepsilon}(K)$ the solution $z(\lambda, \theta, x)$ lies in $D^{\prime}$, we want to compare it with the solution $z\left(\tilde{\lambda}, \theta, x_{0}\right)$, where $x_{0}$ is an arbitrary point of $K$ at a distance of at most $\varepsilon$ from $x$. We want to show that these solutions are $\frac{\beta \delta}{25}$-close for all $\theta \in[0,2 \pi]$. To estimate the distance between two solutions of close differential equations with close initial conditions we use Gronwall's inequality, as stated for our case:

Suppose that

$$
\begin{gathered}
\left|F_{\tilde{\lambda}}(z, \theta)-F_{\lambda}(z, \theta)\right| \leq \alpha \text { and } \\
\left.\left|F_{\tilde{\lambda}}^{\prime}(z, \theta)\right|<L \text { (the derivative is with respect to } z\right)
\end{gathered}
$$

for $(z, \theta) \in D^{\prime}$. If

$$
e^{2 \pi L}(\varepsilon+2 \pi \alpha)<\frac{\beta \delta}{25}
$$

then

$$
\left|z(\lambda, \theta, x)-z\left(\tilde{\lambda}, \theta, x_{0}\right)\right| \leq e^{\theta L}(\varepsilon+2 \pi \alpha) \quad \text { for } \theta \in[0,2 \pi] .
$$

Thus, we need to estimate $L$ and $\alpha$, as well as to check inequality (20). First, we find an $L$ that would make (19) hold:

$$
F_{\tilde{\lambda}}^{\prime}(z, \theta)=\frac{\tilde{\lambda}_{1}+z f_{\tilde{\lambda}}}{1+z g_{\tilde{\lambda}}}+z \frac{f_{\tilde{\lambda}}-\tilde{\lambda}_{1} g_{\tilde{\lambda}}}{\left(1+z g_{\tilde{\lambda}}\right)^{2}}
$$

It follows from Section 1 that $\left|\tilde{\lambda}_{1}\right| \leq \frac{10}{\delta}$. We also have $\left|f_{\tilde{\lambda}}\right| \leq 10,\left|g_{\tilde{\lambda}}\right| \leq 10$ and

$$
|z|<\frac{1}{\delta}+\frac{\beta \delta}{25}<\frac{10}{9 \delta}
$$

To estimate the denominators in the expression for $F_{\tilde{\lambda}}^{\prime}$, consider two cases. If $\left|g_{\tilde{\lambda}}(\theta)\right|<$ $\frac{4 \delta}{5}$, then $\left|1+z g_{\tilde{\lambda}}\right|>1-8 / 9=1 / 9$. If $\left|g_{\tilde{\lambda}}(\theta)\right| \geq \frac{4 \delta}{5}$, then, for some point $z_{0}$ such that $\left(z_{0}, \theta\right) \in D$ and $\left|z-z_{0}\right|<\frac{\beta \delta}{25}$,

$$
\left|\frac{1}{g_{\tilde{\lambda}}}+z_{0}\right| \geq \beta
$$

If $g_{\tilde{\lambda}}(\theta) \leq-\frac{4 \delta}{5}$, this follows from Proposition 2, and if $g_{\tilde{\lambda}}(\theta) \geq \frac{4 \delta}{5}$, it follows from the inequality $\left|g_{\tilde{\lambda}}(\theta)\right| \leq 10$. We now have

$$
\left|1+z g_{\tilde{\lambda}}\right| \geq\left|\frac{1}{g_{\tilde{\lambda}}}+z_{0}\right|\left|g_{\tilde{\lambda}}\right|-\left|z-z_{0}\right|\left|g_{\tilde{\lambda}}\right| \geq \frac{4 \beta \delta}{5}-10 \frac{\beta \delta}{25}=\frac{2 \beta \delta}{5} .
$$

It follows that

$$
L<\frac{10^{3}}{\beta^{2} \delta^{3}}
$$


Now we find an $\alpha$ that would make (18) hold:

$$
\begin{aligned}
\left|F_{\tilde{\lambda}}(z, \theta)-F_{\lambda}(z, \theta)\right| & =\left|z \frac{\left(\tilde{\lambda}_{1}+z f_{\tilde{\lambda}}\right)\left(1+z g_{\lambda}\right)-\left(\lambda_{1}+z f_{\lambda}\right)\left(1+z g_{\tilde{\lambda}}\right)}{\left(1+z g_{\tilde{\lambda}}\right)\left(1+z g_{\lambda}\right)}\right| \\
& =|z|\left|\frac{N}{\left(1+z g_{\tilde{\lambda}}\right)\left(1+z g_{\lambda}\right)}\right|
\end{aligned}
$$

where $N$ denotes the numerator of the corresponding fraction. Since $\lambda \in \Delta_{\omega}(\tilde{\lambda})$, we have $\left|f_{\tilde{\lambda}}-f_{\lambda}\right|<10 \gamma$ and $\left|g_{\tilde{\lambda}}-g_{\lambda}\right|<10 \gamma$. As we already saw, $\left|1+z g_{\tilde{\lambda}}\right|>\frac{2 \beta \delta}{5}$, and therefore

$$
\left|1+z g_{\lambda}\right| \geq\left|1+z g_{\tilde{\lambda}}\right|-\frac{10}{9 \delta}\left|g_{\tilde{\lambda}}-g_{\lambda}\right| \geq \frac{2 \beta \delta}{5}-\frac{10}{9 \delta} \cdot 10 \gamma \geq \frac{\beta \delta}{5},
$$

where the last inequality holds because of the choice of $\gamma$ (see (14)). Now we estimate $N$ :

$$
\begin{aligned}
|N|= & \mid\left(\tilde{\lambda}_{1}-\lambda_{1}\right)+z\left(f_{\tilde{\lambda}}-f_{\lambda}\right)+z\left(\tilde{\lambda}_{1}\left(g_{\lambda}-g_{\tilde{\lambda}}\right)\right. \\
& \left.-g_{\tilde{\lambda}}\left(\lambda_{1}-\tilde{\lambda}_{1}\right)\right)+z^{2}\left(f_{\tilde{\lambda}}\left(g_{\lambda}-g_{\tilde{\lambda}}\right)-g_{\tilde{\lambda}}\left(f_{\lambda}-f_{\tilde{\lambda}}\right)\right) \mid \\
< & 382 \gamma \delta^{-2} .
\end{aligned}
$$

Finally,

To verify (20), notice that

$$
\alpha \leq \frac{9 \cdot 10^{4} \gamma}{2 \pi \beta^{2} \delta^{5}}
$$

$$
e^{2 \pi L}(\varepsilon+2 \pi \alpha)<e^{2 \pi L} \frac{10^{5} \gamma}{\beta^{2} \delta^{5}} \leq \frac{\beta \delta}{25}
$$

because of the choice of $\varepsilon$ and $\gamma$. Thus, inequality (21) holds, whence

$$
\left|z(\lambda, \theta, x)-z\left(\tilde{\lambda}, \theta, x_{0}\right)\right| \leq e^{\theta L}(\varepsilon+2 \pi \alpha)<\frac{\beta \delta}{25} .
$$

Since for all $\theta \in[0,2 \pi]$ the solution $z\left(\tilde{\lambda}, \theta, x_{0}\right)$ lies in $D$, the solution $z(\lambda, \theta, x)$ lies in $D^{\prime}$. This finishes the proof of Lemma 2 .

\section{Proof of Lemma 3}

In this section we shall prove Lemma 3 on a suitable polydisk $\Delta_{\rho}(\xi)$ centered at $\xi \in \Sigma$ for the Bautin ideal $I_{\rho}(\xi)$ of $D_{\lambda}(x)$. Recall that Lemma 3 asserts the existence of such a polydisk with given conditions on its polyradius and estimates the growth constant for the ideal $I_{\rho}(\xi)$ with canonical generators.

We know that the Bautin ideal $I(\Sigma)$ is of index 7. Moreover, we can exclude the even-numbered coefficients from the first seven coefficients $g_{1}(\lambda), \ldots, g_{7}(\lambda)$ of the Taylor series for $D_{\lambda}(x)$, and the remaining coefficients $g_{1}, g_{3}, g_{5}, g_{7}$ generate $I(\Sigma)$ (see, for example, [7]). An important role in the proof of Lemma 3 is played by a connection between canonical generators of the Bautin ideal of $D_{\lambda}(x)$ and the Dulac generators $f_{1}(\lambda), \ldots, f_{4}(\lambda)$ :

$$
\left(\begin{array}{l}
g_{1} \\
g_{3} \\
g_{5} \\
g_{7}
\end{array}\right)=\left(\begin{array}{cccc}
a_{11} & 0 & 0 & 0 \\
a_{21} & a_{22} & 0 & 0 \\
a_{31} & a_{32} & a_{33} & 0 \\
a_{41} & a_{42} & a_{43} & a_{44}
\end{array}\right)\left(\begin{array}{l}
f_{1} \\
f_{2} \\
f_{3} \\
f_{4}
\end{array}\right)=A \cdot\left(\begin{array}{l}
f_{1} \\
f_{2} \\
f_{3} \\
f_{4}
\end{array}\right) .
$$

The entries $a_{i j}$ of the matrix $A$ depend analytically on $\lambda$ and for $\lambda_{1}=0$ are polynomials in $\lambda_{2}, \ldots, \lambda_{6}$.

The proof of Lemma 3 consists of three steps. First, for any point $\xi \in \mathbb{C}^{6}$ we shall find, in Subsection 6.1, a marked polydisk. For the definition of a marked polydisk, see below. Such a polydisk has certain properties which are stated in terms of its intersection with 
the loci of irreducible factors of the polynomials $f_{1}, \ldots, f_{4}$ in $\mathbb{C}^{6}$. Then, in Subsection 6.2. we shall find a growth constant for the ideal $I_{\rho}(\xi)$ with Dulac generators $\left\{f_{i}\right\}$ in a marked polydisk centered in the locus. In particular, we shall show that the marked polydisk is suitable. Finally, in Subsection 6.3, we shall obtain estimates on the entries of $A$ and recalculate the growth constant for the canonical generators $\left\{g_{1}, g_{3}, g_{5}, g_{7}\right\}$.

6.1. Constructing a marked polydisk. Later on we shall make use of the following simple result from one-dimensional complex analysis.

Claim 1. Suppose $w \in \mathbb{C}$ and $R>0$. Then either $|\sqrt{z}|<2 \sqrt{R}$ for all $z \in \Delta_{R}(w)$ or one can choose a single-valued branch of $\sqrt{\cdot}$ in $\Delta_{R}(w)$ such that

$$
\sqrt{z} \in \Delta_{\sqrt{R}}(\sqrt{w})
$$

for all $z \in \Delta_{R}(w)$.

Proof. Consider first the case $|w| \leq R$. Then $|z|<2 R$ and $|\sqrt{z}|<\sqrt{2 R}<2 \sqrt{R}$ for any choice of $\sqrt{z}$.

Now consider the case $|w|>R$. Then one can choose a branch of $\sqrt{\cdot}$ in $\Delta_{R}(w)$ and for any $z \in \Delta_{R}(w)$ the angle between $\sqrt{z}$ and $\sqrt{w}$ will be sharp. It follows that

$$
|\sqrt{w}+\sqrt{z}| \geq|\sqrt{w}| \geq \sqrt{R} .
$$

Therefore,

as was claimed.

$$
|\sqrt{w}-\sqrt{z}| \leq \frac{|w-z|}{|\sqrt{w}+\sqrt{z}|} \leq \frac{R}{\sqrt{R}}=\sqrt{R}
$$

Let $\Omega$ be the set of irreducible factors of the polynomials $f_{1}, \ldots, f_{4}$,

$$
\Omega=\left\{\lambda_{1}, \lambda_{2}, \lambda_{4}, \lambda_{5}, p_{6}, p_{4}, p_{2}\right\}
$$

and $\Gamma_{h}$ the locus of the polynomial $h$ in $\mathbb{C}^{6}$,

$$
\Gamma_{h}=\left\{\lambda \in \mathbb{C}^{6} \mid h(\lambda)=0\right\} .
$$

For $h=\lambda_{1}$ (respectively, $\lambda_{2}, \lambda_{4}, \lambda_{5}, p_{6}, p_{4}$ ) let $\pi_{h}: \mathbb{C}^{6} \rightarrow \Gamma_{h}$ be the projection to $\Gamma_{h}$ along $\lambda_{1}$ (respectively, along $\lambda_{2}, \lambda_{4}, \lambda_{5}, \lambda_{6}, \lambda_{4}$ ).

Lemma 4 (on a marked polydisk). For arbitrary $R \in(0,1)$ and any point $\xi \in \mathbb{C}^{6}$ with coordinates $\left|\xi_{i}\right| \leq 2$ there is a polyradius $\rho=\left(\rho_{1}, \ldots, \rho_{6}\right)$ such that

$$
R \leq \rho_{i} \leq 999 \sqrt{R}
$$

for all components $\rho_{i}$ of $\rho$, and for $\lambda \in \Delta_{\rho}(\xi)$ we have:

1) for all $h \in \Omega$, except $p_{2}$, we have $|h| \geq R$ on the skeleton of $\Delta_{\rho}(\xi)$; for $p_{2}$ we have $\left|p_{2}\right| \geq R^{2}$;

2) for any $h \in \Omega$, except $p_{2}$, either $|h(\lambda)| \geq R$ in $\Delta_{\rho}(\xi)$ or $\Delta_{\rho}(\xi) \cap \Gamma_{h} \neq \emptyset$, and in the latter case, $\pi_{h}\left(\Delta_{\rho}(\xi)\right)=\Delta_{\rho}(\xi) \cap \Gamma_{h}$;

$3)$ either $\left|p_{2}(\lambda)\right| \geq R^{2}$ in $\Delta_{\rho}(\xi)$ or for any $\lambda=\left(\lambda_{1}, \ldots, \lambda_{6}\right) \in \Delta_{\rho}(\xi)$ the point $\left(\lambda_{1}, \sqrt{\lambda_{6}\left(\lambda_{3}-2 \lambda_{6}\right)}, \lambda_{3}, \ldots, \lambda_{6}\right)$ belongs to $\Delta_{\rho}(\xi)$ for one of the two possible values of $\sqrt{\lambda_{6}\left(\lambda_{3}-2 \lambda_{6}\right)}$;

4) if $\xi_{4}=0$ and $\Delta_{\rho}(\xi) \cap \Gamma_{p_{4}} \neq \emptyset$, then $\Delta_{\rho}(\xi) \cap \Gamma_{p_{6}} \neq \emptyset$;

5) if $\xi_{2}=0$ and $\Delta_{\rho}(\xi) \cap \Gamma_{p_{2}} \neq \emptyset$, then $\Delta_{\rho}(\xi) \cap \Gamma_{q} \neq \emptyset$, where $q=\lambda_{6}\left(\lambda_{3}-2 \lambda_{6}\right)$ and $\Gamma_{q}$ is the locus of $q$.

Proof. Suppose $\xi=\left(\xi_{1}, \ldots, \xi_{6}\right)$. We shall describe how to choose the components $\rho_{1}, \ldots, \rho_{6}$ of the polyradius $\rho$. 
Choosing $\rho_{1}$. Consider the circles $\partial \Delta_{R}\left(\xi_{1}\right)$ and $\partial \Delta_{3 R}\left(\xi_{1}\right)$. At least one of them does not intersect the disk $\Delta_{R}(0)$. Let $\rho_{1}$ be the radius of that circle. Now

$$
\left|\lambda_{1}\right| \geq R \text { on } \partial \Delta_{\rho_{1}}\left(\xi_{1}\right)
$$

and either

a) $\lambda_{1}=0 \in \Delta_{\rho_{1}}\left(\xi_{1}\right)$

or

b) $\left|\lambda_{1}\right| \geq R$ on $\overline{\Delta_{\rho_{1}}\left(\xi_{1}\right)}$.

Choosing $\rho_{5}$. We choose $\rho_{5}$ the same way as $\rho_{1}$. We then have either $\rho_{5}=R$ or $\rho_{5}=3 R$. Also

$$
\left|\lambda_{5}\right| \geq R \text { on } \partial \Delta_{\rho_{5}}\left(\xi_{5}\right)
$$

and either

c) $\lambda_{5}=0 \in \Delta_{\rho_{5}}\left(\xi_{5}\right)$

or

d) $\left|\lambda_{5}\right| \geq R$ on $\overline{\Delta_{\rho_{5}}\left(\xi_{5}\right)}$.

Choosing $\rho_{3}$. Set $\rho_{3}=R$.

Choosing $\rho_{6}$. Consider the circles $\partial \Delta_{R}\left(\xi_{6}\right), \partial \Delta_{5 R}\left(\xi_{6}\right), \partial \Delta_{9 R}\left(\xi_{6}\right)$, and $\partial \Delta_{13 R}\left(\xi_{6}\right)$. At least three of them do not intersect $\Delta_{2 R}(0)$, three do not intersect $\Delta_{2 R}\left(\xi_{3}\right)$, and three do not intersect $\Delta_{3 R / 2}\left(\frac{\xi_{3}}{2}\right)$. Therefore, at least one circle does not intersect any of these sets. Let $\rho_{6}$ be its radius.

In view of our choices for $\rho_{6}$ and $\rho_{3}$, we have

$$
\left|\lambda_{3}-\lambda_{6}\right| \geq R \text { for }\left(\lambda_{3}, \lambda_{6}\right) \in \partial \Delta_{\rho_{3}}\left(\xi_{3}\right) \times \partial \Delta_{\rho_{6}}\left(\xi_{6}\right)
$$

and either

e) for any $\lambda_{3} \in \Delta_{\rho_{3}}\left(\xi_{3}\right)$ the point $\left(\lambda_{3}, \lambda_{3}\right)$ belongs to $\Delta_{\rho_{3}}\left(\xi_{3}\right) \times \Delta_{\rho_{6}}\left(\xi_{6}\right)$ or

f) $\left|\lambda_{3}-\lambda_{6}\right| \geq R$ on $\overline{\Delta_{\rho_{3}}\left(\xi_{3}\right) \times \Delta_{\rho_{6}}\left(\xi_{6}\right)}$.

Similarly, it is easy to see that

$$
\left|\lambda_{6}\right| \geq 2 R \text { and }\left|\lambda_{6}-\frac{\lambda_{3}}{2}\right| \geq R \text { for }\left(\lambda_{3}, \lambda_{6}\right) \in \partial \Delta_{\rho_{3}}\left(\xi_{3}\right) \times \partial \Delta_{\rho_{6}}\left(\xi_{6}\right),
$$

i.e., $|q|=\left|\lambda_{6}\left(2 \lambda_{6}-\lambda_{3}\right)\right| \geq 4 R^{2}$ on the product of those circles, and either

g) for any $\lambda_{3} \in \Delta_{\rho_{3}}\left(\xi_{3}\right)$ the polynomial $q$ vanishes at some point of $\Delta_{\rho_{6}}\left(\xi_{6}\right)$ or

h) $|q| \geq 4 R^{2}$ on $\overline{\Delta_{\rho_{3}}\left(\xi_{3}\right) \times \Delta_{\rho_{6}}\left(\xi_{6}\right)}$.

Choosing $\rho_{4}$. Suppose that $\xi_{4}=0$. If $\left|p_{4}\right| \geq R$ for arbitrary $\lambda_{4} \in \overline{\Delta_{R}\left(\xi_{4}\right)}$ and $\left(\lambda_{3}, \lambda_{6}\right) \in$ $\overline{\Delta_{\rho_{3}}\left(\xi_{3}\right) \times \Delta_{\rho_{6}}\left(\xi_{6}\right)}$, then we set $\rho_{4}=R$. We then also have

i) $\left|\lambda_{4}\right| \geq R$ on $\partial \Delta_{\rho_{4}}\left(\xi_{4}\right)$ and

j) $\left|p_{4}\right| \geq R$ for $\left(\lambda_{3}, \lambda_{4}, \lambda_{6}\right) \in \overline{\Delta_{\rho_{3}}\left(\xi_{3}\right) \times \Delta_{\rho_{4}}\left(\xi_{4}\right) \times \Delta_{\rho_{6}}\left(\xi_{6}\right)}$.

In the remaining cases, we choose $\rho_{4}$ such that $\rho_{4} \geq R$. With this choice, if $\xi_{4}=0$ but $\left|p_{4}\right|<R$ for some $\lambda_{4} \in \overline{\Delta_{R}\left(\xi_{4}\right)}$ and $\left(\lambda_{3}, \lambda_{6}\right) \in \overline{\Delta_{\rho_{3}}\left(\xi_{3}\right) \times \Delta_{\rho_{6}}\left(\xi_{6}\right)}$, then

$$
\left|5\left(\lambda_{3}-\lambda_{6}\right)\right| \leq\left|p_{4}\right|+\left|\lambda_{4}\right|<2 R \Rightarrow\left|p_{6}\right|<R,
$$

and therefore, assertion e) is true in this case. Thus we have shown that for $\xi_{4}=0$ we have either j) or e), which implies assertion 4) of the lemma. 
Suppose now that $\xi_{4}$ is arbitrary and that $\rho_{4}$ has not yet been chosen. Notice that

$$
-5\left(\lambda_{3}-\lambda_{6}\right) \in \Delta_{70 R}\left(-5\left(\xi_{3}-\xi_{6}\right)\right)
$$

for $\lambda_{3} \in \Delta_{\rho_{3}}\left(\xi_{3}\right)$ and $\lambda_{6} \in \Delta_{\rho_{6}}\left(\xi_{6}\right)$. Consider the circles $\partial \Delta_{R}\left(\xi_{4}\right), \partial \Delta_{143 R}\left(\xi_{4}\right)$, and $\partial \Delta_{285 R}\left(\xi_{4}\right)$. At least two of them do not intersect $\Delta_{71 R}\left(-5\left(\xi_{3}-\xi_{6}\right)\right)$, and at least two do not intersect $\Delta_{R}(0)$. Therefore, at least one of them does not intersect any of those sets. Let $\rho_{4}$ be its radius. We conclude that

$$
\left|\lambda_{4}\right| \geq R \text { on } \partial \Delta_{\rho_{4}}\left(\xi_{4}\right)
$$

and

$$
\left|\lambda_{4}+5\left(\lambda_{3}-\lambda_{6}\right)\right| \geq R \text { for }\left(\lambda_{3}, \lambda_{4}, \lambda_{6}\right) \in \partial \Delta_{\rho_{3}}\left(\xi_{3}\right) \times \partial \Delta_{\rho_{4}}\left(\xi_{4}\right) \times \partial \Delta_{\rho_{6}}\left(\xi_{6}\right) .
$$

In this case, either

k) $\lambda_{4}=0 \in \Delta_{\rho_{4}}\left(\xi_{4}\right)$

or

l) $\left|\lambda_{4}\right| \geq R$ on $\overline{\Delta_{\rho_{4}}\left(\xi_{4}\right)}$

and, moreover, either

m) for any $\left(\lambda_{3}, \lambda_{6}\right) \in \Delta_{\rho_{3}}\left(\xi_{3}\right) \times \Delta_{\rho_{6}}\left(\xi_{6}\right)$ the point $\left(-5\left(\lambda_{3}-\lambda_{6}\right), \lambda_{3}, \lambda_{6}\right)$ belongs to $\Delta_{\rho_{4}}\left(\xi_{4}\right) \times \Delta_{\rho_{3}}\left(\xi_{3}\right) \times \Delta_{\rho_{6}}\left(\xi_{6}\right)$

$$
\text { or }
$$

n) $\left|\lambda_{4}+5\left(\lambda_{3}-\lambda_{6}\right)\right| \geq R$ on $\overline{\Delta_{\rho_{4}}\left(\xi_{4}\right) \times \Delta_{\rho_{3}}\left(\xi_{3}\right) \times \Delta_{\rho_{6}}\left(\xi_{6}\right)}$.

Choosing $\rho_{2}$. Suppose that $\xi_{2}=0$. If $\left|p_{2}\right| \geq R^{2}$ for arbitrary $\lambda_{2} \in \overline{\Delta_{R}\left(\xi_{2}\right)}$ and $\left(\lambda_{3}, \lambda_{6}\right) \in$ $\overline{\Delta_{\rho_{3}}\left(\xi_{3}\right) \times \Delta_{\rho_{6}}\left(\xi_{6}\right)}$, set $\rho_{2}=R$. We then have

o) $\left|\lambda_{2}\right| \geq R$ on $\partial \Delta_{\rho_{2}}(0)$ and

p) $\left|p_{2}\right| \geq R^{2}$ for $\left(\lambda_{2}, \lambda_{3}, \lambda_{6}\right) \in \overline{\Delta_{\rho_{2}}\left(\xi_{2}\right) \times \Delta_{\rho_{3}}\left(\xi_{3}\right) \times \Delta_{\rho_{6}}\left(\xi_{6}\right)}$.

In the remaining cases choose $\rho_{2}$ such that $\rho_{2} \geq R$. With such a choice, if $\xi_{2}=0$ but $\left|p_{2}\right|<R^{2}$ for some $\lambda_{2} \in \overline{\Delta_{R}\left(\xi_{2}\right)}$ and $\left(\lambda_{3}, \lambda_{6}\right) \in \overline{\Delta_{\rho_{3}}\left(\xi_{3}\right) \times \Delta_{\rho_{6}}\left(\xi_{6}\right)}$, then

$$
|q|=\left|\lambda_{6}\left(\lambda_{3}-2 \lambda_{6}\right)\right| \leq\left(\sqrt{\left|p_{2}\right|}+\left|\lambda_{2}\right|\right)^{2}<4 R^{2},
$$

and therefore, assertion g) is true in this case. Thus we have shown that for $\xi_{2}=0$ we have either $\mathrm{p}$ ) or $\mathrm{g}$ ), which implies assertion 5) of the lemma.

Suppose now that $\xi_{2}$ is arbitrary and $\rho_{2}$ has not yet been chosen. Notice that, for $\lambda_{3} \in \Delta_{\rho_{3}}\left(\xi_{3}\right)$ and $\lambda_{6} \in \Delta_{\rho_{6}}\left(\xi_{6}\right)$,

$$
\lambda_{6}\left(\lambda_{3}-2 \lambda_{6}\right) \in \Delta_{483 R}\left(\xi_{6}\left(\xi_{3}-2 \xi_{6}\right)\right) .
$$

Indeed, if $\lambda_{3}=\xi_{3}+d_{3}$ and $\lambda_{6}=\xi_{6}+d_{6}$, then

$$
\lambda_{6}\left(\lambda_{3}-2 \lambda_{6}\right)=\xi_{6}\left(\xi_{3}-2 \xi_{6}\right)+d_{6}\left(\xi_{3}-2 \xi_{6}\right)+\xi_{6}\left(d_{3}-2 d_{6}\right)+d_{6}\left(d_{3}-2 d_{6}\right),
$$

and (24) follows from the inequalities $\left|\xi_{i}\right| \leq 2,\left|d_{3}\right| \leq R,\left|d_{6}\right| \leq 13 R$ and $R \leq 1$. Claim 1 together with (24) implies that, for $\lambda_{3} \in \Delta_{\rho_{3}}\left(\xi_{3}\right)$ and $\lambda_{6} \in \Delta_{\rho_{6}}\left(\xi_{6}\right)$,

$$
\sqrt{\lambda_{6}\left(\lambda_{3}-2 \lambda_{6}\right)} \in \Delta_{2 \sqrt{483 R}}(w) \subset \Delta_{44 \sqrt{R}}(w),
$$

where $w=0$ or $w=\sqrt{\xi_{6}\left(\xi_{3}-2 \xi_{6}\right)}$. Notice that

$$
p_{2}=\lambda_{6}\left(\lambda_{3}-2 \lambda_{6}\right)-\lambda_{2}^{2}=\left(\sqrt{\lambda_{6}\left(\lambda_{3}-2 \lambda_{6}\right)}-\lambda_{2}\right)\left(\sqrt{\lambda_{6}\left(\lambda_{3}-2 \lambda_{6}\right)}+\lambda_{2}\right) .
$$

Consider the four circles $\partial \Delta_{\sqrt{R}}\left(\xi_{2}\right), \partial \Delta_{91 \sqrt{R}}\left(\xi_{2}\right), \partial \Delta_{181 \sqrt{R}}\left(\xi_{2}\right)$, and $\partial \Delta_{271 \sqrt{R}}\left(\xi_{2}\right)$. At least three of them do not intersect $\Delta_{45 \sqrt{R}}(w)$. Three do not intersect $\Delta_{45 \sqrt{R}}(-w)$ and 
three do not intersect $\Delta_{R}(0)$. Therefore, at least one circle does not intersect any of these sets. Let $\rho_{2}$ be its radius. Then

$$
\left|\lambda_{2}\right| \geq R \text { on } \partial \Delta_{\rho_{2}}\left(\xi_{2}\right)
$$

and

$$
\begin{gathered}
\left|\lambda_{6}\left(\lambda_{3}-2 \lambda_{6}\right)-\lambda_{2}^{2}\right| \geq \sqrt{R} \sqrt{R}=R \geq R^{2} \\
\text { for }\left(\lambda_{2}, \lambda_{3}, \lambda_{6}\right) \in \partial \Delta_{\rho_{2}}\left(\xi_{2}\right) \times \partial \Delta_{\rho_{3}}\left(\xi_{3}\right) \times \partial \Delta_{\rho_{6}}\left(\xi_{6}\right) .
\end{gathered}
$$

In this case, either

q) $\lambda_{2}=0 \in \Delta_{\rho_{2}}\left(\xi_{2}\right)$

or

r) $\left|\lambda_{2}\right| \geq R$ on $\overline{\Delta_{\rho_{2}}\left(\xi_{2}\right)}$

and, moreover, either

s) for any $\left(\lambda_{3}, \lambda_{6}\right) \in \Delta_{\rho_{3}}\left(\xi_{3}\right) \times \Delta_{\rho_{6}}\left(\xi_{6}\right)$, at least one of the points $\left( \pm \sqrt{\lambda_{6}\left(\lambda_{3}-2 \lambda_{6}\right)}\right.$, $\left.\lambda_{3}, \lambda_{6}\right)$ belongs to $\Delta_{\rho_{2}}\left(\xi_{2}\right) \times \Delta_{\rho_{3}}\left(\xi_{3}\right) \times \Delta_{\rho_{6}}\left(\xi_{6}\right)$ (this happens when one of the disks $\Delta_{45 \sqrt{R}}( \pm w)$ is contained in $\left.\Delta_{\rho_{2}}\left(\xi_{2}\right)\right)$ or

t) $\left|p_{2}(\lambda)\right| \geq R$ on $\overline{\Delta_{\rho_{2}}\left(\xi_{2}\right) \times \Delta_{\rho_{3}}\left(\xi_{3}\right) \times \Delta_{\rho_{6}}\left(\xi_{6}\right)}$ (this happens when none of the disks $\Delta_{45 \sqrt{R}}( \pm w)$ intersects $\left.\Delta_{\rho_{2}}\left(\xi_{2}\right)\right)$.

Thus, we have chosen $\rho=\left(\rho_{1}, \ldots, \rho_{6}\right)$. Since $R<\sqrt{R}$ when $R<1$, it is easy to check that (23) holds for $\Delta_{\rho}(\xi)$. Assertion 1) of the lemma now follows from the obtained estimates on the $\rho_{i}$. Assertion 2) follows from a) - r) above. Assertion 3) follows from s) and t). Assertions 4) and 5) were proved earlier.

Corollary 1. Lemma 4 remains true if in 1)-5) nonstrict inequalities are replaced by strict ones, and (23) is replaced by

$$
R<\rho_{i}<1000 \sqrt{R}
$$

Proof. Suppose $R \in(0,1)$ and $\varepsilon>0$ is so small that $R+\varepsilon \in(0,1)$ and $999 \sqrt{R+\varepsilon}<$ $1000 \sqrt{R}$. Apply Lemma 4 with $R$ replaced by $R+\varepsilon$. For the resulting polyradius $\rho$, all assertions of the corollary are true.

Definition 9. Suppose $R \in(0,1)$ and $\xi \in \mathbb{C}^{6}$ is a point with coordinates $\left|\xi_{i}\right| \leq 2$. A polydisk $\Delta_{\rho}(\xi)$ satisfying the conditions of Corollary 1 is called a marked $R$-polydisk or, if $R$ is not important, simply a marked polydisk.

From the definition of a marked polydisk we immediately have

Corollary 2. The property of a polydisk to be a marked R-polydisk is preserved under small changes of its polyradius.

We shall later show (Lemma 7) that a marked polydisk is suitable for the Bautin ideal. Here is another important corollary of Lemma 4, which will be used in the proof of Lemma 7.

Corollary 3. Suppose $h \in \Omega$ is an irreducible factor of the $f_{i}$. If $\Gamma_{h}$ intersects a marked polydisk $\Delta_{\rho}(\xi)$ centered at $\xi \in \Sigma$, then $\Gamma_{h}$ has a nonempty intersection with $\Sigma$ inside that polydisk.

Proof. If $h \in\left\{\lambda_{1}, \lambda_{2}, \lambda_{4}, \lambda_{5}, p_{6}\right\}$ and $\Gamma_{h} \cap \Delta_{\rho}(\xi) \neq \emptyset$, then Lemma 4, 2) and formulas (4) and (5) for the $f_{i}$ show that $\pi_{h}(\xi) \in \Sigma \cap \Gamma_{h} \cap \Delta_{\rho}(\xi)$.

If $\Gamma_{p_{4}} \cap \Delta_{\rho}(\xi) \neq \emptyset$ and $\xi_{4} \neq 0$, then $\pi_{p_{4}}(\xi) \in \Sigma \cap \Gamma_{p_{4}} \cap \Delta_{\rho}(\xi)$.

If $\Gamma_{p_{4}} \cap \Delta_{\rho}(\xi) \neq \emptyset$ and $\xi_{4}=0$, then Lemma 4 4) shows that $\Gamma_{p_{6}} \cap \Delta_{\rho}(\xi) \neq \emptyset$. Now Lemma 44 2) and formulas (4) and (5) for the $f_{i}$ show that $\pi_{p_{4}} \circ \pi_{p_{6}}(\xi) \in \Sigma \cap \Gamma_{p_{4}} \cap \Delta_{\rho}(\xi)$. 
If $\Gamma_{p_{2}} \cap \Delta_{\rho}(\xi) \neq \emptyset$ and either $\xi_{2} \neq 0$ or $\xi_{2}=0$ together with $p_{6}(\xi)=0$, then we can change the coordinate $\xi_{2}$ of $\xi$ so that the new point would remain inside $\Delta_{\rho}(\xi)$ and would belong to $\Gamma_{p_{2}}$. This follows from Lemma 4, 3). Furthermore, as is easy to check, the expressions for the $f_{i}$ in each of the two cases show that the new point is in $\Sigma$.

It remains to consider the case $\Gamma_{p_{2}} \cap \Delta_{\rho}(\xi) \neq \emptyset, \xi_{2}=0$, and $p_{6}(\xi) \neq 0$. Under these assumptions, since $f_{1}(\xi)=f_{2}(\xi)=0$, we have $\xi_{1}=\xi_{5}=0$. Changing the coordinates $\xi_{3}$ and $\xi_{6}$ of $\xi$ we can keep the new point inside $\Delta_{\rho}(\xi)$ and make $q$ vanish at it. This follows from Lemma 4, 5). As $\xi_{2}=0$, at that point $p_{2}=0$. Using the expressions for the $f_{i}$ we now easily check that the new point is in $\Gamma_{p_{2}} \cap \Sigma$.

6.2. Growth constant for the Bautin ideal $I_{\rho}(\xi)$ and Dulac generators. Consider the Dulac generators $f_{1}(\lambda), \ldots, f_{4}(\lambda)$ given by (44) and (5).

Lemma 5. Suppose $\xi \in \Sigma, 0<R<10^{-7}$ and $\Delta_{\rho}(\xi)$ is a marked $R$-polydisk. If $f \in$ $O\left(\Delta_{\rho}(\xi)\right)$ and the germ of $f$ on $\Sigma$ belongs to $I(\Sigma)$, then there are functions $a_{1}, \ldots, a_{4} \in$ $O\left(\Delta_{\rho}(\xi)\right)$ such that

$$
f=a_{1} f_{1}+a_{2} f_{2}+a_{3} f_{3}+a_{4} f_{4},
$$

and for some $\varepsilon>0$,

$$
\left\|a_{i}\right\|_{\tilde{\rho}, \xi} \leq C\|f\|_{\tilde{\rho}, \xi}, \text { where } C=\frac{3}{R^{7}}
$$

in any polydisk $\Delta_{\tilde{\rho}}(\xi) \subset \Delta_{\rho}(\xi)$ such that $\|\tilde{\rho}-\rho\|<\varepsilon$.

To prove this lemma we need some preliminary results from the theory of functions of several complex variables.

Proposition 3. Suppose $U$ is a domain in $\mathbb{C}^{n}$, functions $f$ and $g$ are holomorphic in $U$, $\Gamma=\{t \in U \mid g(t)=0\}$ is the zero set of $g$ in $U$, and $\Gamma^{\prime}=\{t \in \Gamma \mid \operatorname{grad} g=0\}$ the subset of $\Gamma$ where all partials of $g$ vanish. Set $S=\Gamma-\Gamma^{\prime} \subset U$. Suppose that $S$ is connected and $\bar{S} \supset \Gamma$. If $\left.f\right|_{\Gamma \cap V}=0$ in some subdomain $V \subset U$ such that $V \cap \Gamma \neq \emptyset$, then $\left.f\right|_{\Gamma}=0$.

Proof. By the implicit function theorem, $S$ is an analytic manifold. The holomorphic function $f$ is identically zero on the open subset $S \cap V$. Since $S$ is connected, by the uniqueness theorem, we have $\left.f\right|_{S}=0$. As $\bar{S} \supset \Gamma$, we have $\left.f\right|_{\Gamma}=0$ by continuity.

Proposition 4. Suppose that $U$ is a domain in $\mathbb{C}^{n}$, functions $g, f$ belong to $O(U)$, $\Gamma=\{t \in U \mid g(t)=0\}$, and the germ of $g$ is irreducible at any point of $U$. If $\left.f\right|_{\Gamma}=0$, then $f$ is divisible by $g$ in $U$; in other words, there is a function $h \in O(U)$ such that $f=g h$.

For a proof, see [4, Chapter II, Theorem 6].

The next result now follows easily from Propositions 3 and 4

Proposition 5. Suppose $U$ is a domain in $\mathbb{C}^{n}$ and functions $f$ and $g$ are holomorphic in U. Suppose $\Gamma, \Gamma^{\prime}, S$ are defined as in Proposition 3 , the set $S$ is connected and $\bar{S} \supset \Gamma$. If $\left.f\right|_{\Gamma \cap V}=0$ in some subdomain $V \subset U$ such that $V \cap \Gamma \neq \emptyset$, and the germ of $g$ is irreducible at any point of $U$, then $f$ is divisible by $g$ in $U$.

At the beginning of Subsection 6.1 we defined irreducible loci $\Gamma_{h}$ and projections $\pi_{h}: \mathbb{C}^{6} \rightarrow \Gamma_{h}$ for all $h \in \Omega$, except $p_{2}$. In what follows it would be convenient to use the following notation:

and

$$
\pi_{1}:=\pi_{\lambda_{1}}, \quad \pi_{5}:=\pi_{\lambda_{5}}, \quad \pi_{4}:=\pi_{p_{4}}
$$

$$
\Gamma_{1}:=\Gamma_{\lambda_{1}}, \quad \Gamma_{5}:=\Gamma_{\lambda_{5}}, \quad \Gamma_{4}:=\Gamma_{p_{4}} .
$$

The next proposition is the main tool in the proof of Lemma 5. It would allow us to find the coefficients $a_{i}$ in decomposition (25). 
Proposition 6. Suppose $\xi \in \mathbb{C}^{6}$ is an arbitrary point with coordinates $\left|\xi_{i}\right| \leq 2, \Delta_{\rho}(\xi)$ a marked polydisk, and $f \in O\left(\Delta_{\rho}(\xi)\right)$. For any $i \in\{1,4,5\}$ such that $\Gamma_{i} \cap \Delta_{\rho}(\xi) \neq \emptyset$, we have the following:

a) the function $f \circ \pi_{i}$ is defined and holomorphic in $\Delta_{\rho}(\xi)$, and $f \circ \pi_{i}$ does not depend on $\lambda_{i}$;

b) $f-f \circ \pi_{i}=0$ on $\Gamma_{i} \cap \Delta_{\rho}(\xi)$;

c) the functions $\frac{f-f \circ \pi_{i}}{\lambda_{i}}($ for $i=1,5)$ and $\frac{f-f \circ \pi_{i}}{p_{4}}($ for $i=4)$ are holomorphic in $\Delta_{\rho}(\xi)$.

Proof. It follows from the properties of a marked polydisk that $\pi_{i}\left(\Delta_{\rho}(\xi)\right) \subset \Delta_{\rho}(\xi)$. a) and b) now follow at once. c) follows from b) because of Proposition 4 .

Decomposition by generators in a sufficiently small marked polydisk (a local result). Now we want to explicitly determine the coefficients in the decomposition of $f \in$ $I(\Sigma)$ by the Dulac generators $f_{1}, f_{2}, f_{3}, f_{4}$ in a sufficiently small marked polydisk centered at $\xi \in \Sigma$.

Lemma 6. Suppose $\xi \in \Sigma$, the function $f(\lambda)$ is holomorphic in a neighborhood of $\xi$ and $f \in I(\Sigma)$. Suppose also that $R$ is so small that in a marked $R$-polydisk $\Delta_{\rho}(\xi)$ the function $f$ can be represented as a sum

$$
f=b_{1} f_{1}+b_{2} f_{2}+b_{3} f_{3}+b_{4} f_{4}
$$

with holomorphic coefficients $b_{i} \in O\left(\Delta_{\rho}(\xi)\right)$. Consider the following three cases:

1) if $\Gamma_{5} \cap \Delta_{\rho}(\xi)=\emptyset$, set

$$
a_{1}=\frac{f-f \circ \pi_{1}}{f_{1}}, \quad a_{2}=\frac{f \circ \pi_{1}}{f_{2}}, \quad a_{3}=0, \quad a_{4}=0 ;
$$

2) if $\Gamma_{5} \cap \Delta_{\rho}(\xi) \neq \emptyset$, but $\Gamma_{4} \cap \Delta_{\rho}(\xi)=\emptyset$, set

$$
a_{1}=\frac{f-f \circ \pi_{1}}{f_{1}}, \quad a_{2}=\frac{f \circ \pi_{1}-f \circ \pi_{1} \circ \pi_{5}}{f_{2}}, \quad a_{3}=\frac{f \circ \pi_{1} \circ \pi_{5}}{f_{3}}, \quad a_{4}=0 ;
$$

3) if $\Gamma_{5} \cap \Delta_{\rho}(\xi) \neq \emptyset$ and $\Gamma_{4} \cap \Delta_{\rho}(\xi) \neq \emptyset$, set

$$
\begin{aligned}
& a_{1}=\frac{f-f \circ \pi_{1}}{f_{1}}, \\
& a_{2}=\frac{f \circ \pi_{1}-f \circ \pi_{1} \circ \pi_{5}}{f_{2}}, \\
& a_{3}=\frac{\lambda_{4} \cdot\left(\frac{f \circ \pi_{1} \circ \pi_{5}}{\lambda_{4}}-\left(\frac{f \circ \pi_{1} \circ \pi_{5}}{\lambda_{4}}\right) \circ \pi_{4}\right)}{f_{3}}, \\
& a_{4}=\frac{\lambda_{4} \cdot\left(\frac{f \circ \pi_{1} \circ \pi_{5}}{\lambda_{4}}\right) \circ \pi_{4}}{f_{4}} .
\end{aligned}
$$

Then in each of these cases the $a_{i}$ are holomorphic in $\Delta_{\rho}(\xi)$ and

$$
f=a_{1} f_{1}+a_{2} f_{2}+a_{3} f_{3}+a_{4} f_{4} .
$$

Proof. If the $a_{i}$ defined by (28)-(30) are holomorphic, then (31) is automatically true. Thus we want to show that the $a_{i}$ are holomorphic in $\Delta_{\rho}(\xi)$. It is easy to check that

$$
\begin{aligned}
f_{1} \circ \pi_{1} & =0, & f_{i} \circ \pi_{1} & =f_{i} & & \text { for } i=2,3,4 ; \\
f_{2} \circ \pi_{5} & =0, & f_{i} \circ \pi_{5} & =f_{i} & & \text { for } i=3,4 ; \\
\left(\frac{f_{3}}{\lambda_{4}}\right) \circ \pi_{4} & =0, & \left(\frac{f_{4}}{\lambda_{4}}\right) \circ \pi_{4} & =\frac{f_{4}}{\lambda_{4}} & &
\end{aligned}
$$


Therefore

$$
\begin{array}{ll}
h_{1}=f \circ \pi_{1} \in\left(f_{2}, f_{3}, f_{4}\right)_{\rho} & \text { in any of cases 1)-3), } \\
h_{2}=h_{1} \circ \pi_{5} \in\left(f_{3}, f_{4}\right)_{\rho} & \text { except in case } 1), \\
h_{3}=\left(\frac{h_{2}}{\lambda_{4}}\right) \circ \pi_{4} \in\left(\frac{f_{4}}{\lambda_{4}}\right)_{\rho} & \text { in case } 3) .
\end{array}
$$

Indeed, the membership of $h_{1}, h_{2}, h_{3}$ in the corresponding ideals follows at once from (27), provided these functions are holomorphic in $\Delta_{\rho}(\xi)$. But $h_{1}$ and $h_{2}$ are defined and holomorphic in $\Delta_{\rho}(\xi)$ by Proposition 6. Moreover, in case 3) we have $h_{2} \in\left(f_{3}, f_{4}\right)_{\rho} \subset$ $\left(\lambda_{4}\right)_{\rho}$, whence $\frac{h_{2}}{\lambda_{4}} \in O\left(\Delta_{\rho}(\xi)\right)$ and therefore $h_{3} \in O\left(\Delta_{\rho}(\xi)\right)$, also by Proposition 6 .

Consider the difference $f-h_{1}$ on $\Delta_{\rho}(\xi)$. By Proposition [6, it is divisible by $f_{1}$, i.e.,

$$
a_{1}=\frac{f-h_{1}}{f_{1}} \in O\left(\Delta_{\rho}(\xi)\right) .
$$

In case 1$)$, as is easy to see, $\left(f_{2}, f_{3}, f_{4}\right)_{\rho}=\left(f_{2}\right)_{\rho}$, and therefore $a_{2}=\frac{h_{1}}{f_{2}} \in O\left(\Delta_{\rho}(\xi)\right)$. Thus the lemma is proved in case 1).

In cases 2) and 3) consider the difference $h_{1}-h_{2} \in\left(f_{2}, f_{3}, f_{4}\right)_{\rho}$. By Proposition 6, it is divisible by $\lambda_{5}$, and it is divisible by $p_{6}$ because of (4). Therefore, it is divisible by $f_{2}$. Hence

$$
a_{2}=\frac{h_{1}-h_{2}}{f_{2}} \in O\left(\Delta_{\rho}(\xi)\right) .
$$

In case 2), as is easy to see, $\left(f_{3}, f_{4}\right)_{\rho}=\left(f_{3}\right)_{\rho}$, and therefore $a_{3}=\frac{h_{2}}{f_{3}} \in O\left(\Delta_{\rho}(\xi)\right)$. This proves the lemma in case 2 ).

In case 3 ) we have $\frac{h_{2}}{\lambda_{4}}-h_{3} \in\left(\frac{f_{3}}{\lambda_{4}}, \frac{f_{4}}{\lambda_{4}}\right)$. This difference is divisible by $p_{4}$, by Proposition [6, and by $\lambda_{2}$ and $p_{6}$ because of (4). Therefore, it is divisible by $\frac{f_{3}}{\lambda_{4}}$ :

$$
a_{3}=\frac{h_{2}-\lambda_{4} h_{3}}{f_{3}} \in O\left(\Delta_{\rho}(\xi)\right) .
$$

Finally, since $h_{3} \in\left(\frac{f_{4}}{\lambda_{4}}\right)_{\rho}$, we have $a_{4}=\frac{\lambda_{4} h_{3}}{f_{4}} \in O\left(\Delta_{\rho}(\xi)\right)$.

Thus, we have shown that (28)-(30) define (in the corresponding cases) holomorphic functions $a_{i}$ in the polydisk $\Delta_{\rho}(\xi)$. The lemma is proved.

\section{Decomposition by generators in a given marked polydisk.}

Lemma 7. Suppose $0<R<1, \xi \in \Sigma$, and $f \in I(\Sigma)$ is holomorphic in the marked $R$ polydisk $\Delta_{\rho}(\xi)$. Define functions $a_{i}$ on the polydisk $\Delta_{\rho}(\xi)$ as in Lemma (66) ((28) -(30)). Then the $a_{i}$ are defined and holomorphic on the entire $\Delta_{\rho}(\xi)$.

Proof. The definitions of $a_{i}$ depend on the intersection of the polydisk $\Delta_{\rho}(\xi)$ with $\Gamma_{5}$ and $\Gamma_{4}$. It follows easily from Proposition 6 that the numerators of the $a_{i}$ are defined everywhere in $\Delta_{\rho}(\xi)$. Hence the $a_{i}$ are meromorphic in $\Delta_{\rho}(\xi)$. That these functions are holomorphic will be proved separately for points in $\Sigma$ and outside $\Sigma$.

The holomorphicity of the $a_{i}$ in a sufficiently small polydisk $\Delta_{\rho(\eta)}(\eta) \subset \Delta_{\rho}(\xi)$ centered at $\eta \in \Delta_{\rho}(\xi) \cap \Sigma$ is proved the same way as in Lemma 6 if the argument is for the case in 1)-3) corresponding to the ambient polydisk $\Delta_{\rho}(\xi)$.

To show the holomorphicity of the $a_{i}$ outside $\Sigma$, take an arbitrary point $\eta \in \Delta_{\rho}(\xi) \backslash \Sigma$ and consider those irreducible components $\Gamma_{h}$ which correspond to the denominators of the $a_{i}$ and pass through $\eta$. If there are no such components, then all denominators in the $a_{i}$ do not vanish at $\eta$, and all the $a_{i}$ are holomorphic at that point. If there are such components, then for each $h \in \Omega$ such that $\eta \in \Gamma_{h}$, as Corollary 3 shows, there is $\eta^{\prime} \in \Gamma_{h} \cap \Sigma \cap \Delta_{\rho}(\xi)$. In a small neighborhood of $\eta^{\prime}$ the $a_{i}$ are holomorphic. Therefore, by 
Proposition 4, the numerator of each $a_{i}$ is divisible by $h$ in the entire marked polydisk $\Delta_{\rho}(\xi)$. Thus, the $a_{i}$ are holomorphic in $\Delta_{\rho}(\xi)$.

An estimate for the growth constant relative to the Dulac generators. In Lemma 7 we already found coefficients $\left\{a_{i}\right\}$ to satisfy (25). Now we want to establish (26), thus finishing the proof of Lemma 5. Suppose a polyradius $\tilde{\rho}<\rho$ is close to $\rho$ so that $\Delta_{\tilde{\rho}}(\xi)$ is also a marked $R$-polydisk. The coefficients $a_{i}$ are given in $\Delta_{\rho}(\xi)$ by (28), (29), or (30). By the maximum principle, it suffices to estimate their moduli on the skeleton of the polydisk $\Delta_{\tilde{\rho}}(\xi)$, where for any $h \in \Omega$, except $p_{2}$, we have $|h|>R$, and for $p_{2}$ we have $\left|p_{2}\right|>R^{2}$. These inequalities yield lower bounds on the denominators of the $a_{i}$. Upper bounds on the numerators can be found from the following inequalities, which hold on the skeleton of $\Delta_{\tilde{\rho}}(\xi)$ if the expressions under the modulus sign in their left-hand sides are summands in the found $\left\{a_{i}\right\}$ :

$$
\begin{aligned}
|f| & \leq\|f\|_{\tilde{\rho}, \xi}, \\
\left|f \circ \pi_{1}\right| & \leq\|f\|_{\tilde{\rho}, \xi}, \\
\left|f \circ \pi_{1} \circ \pi_{5}\right| & \leq\|f\|_{\tilde{\rho}, \xi}, \\
\frac{f \circ \pi_{1} \circ \pi_{5}}{\lambda_{4}} & \leq \frac{\|f\|_{\tilde{\rho}, \xi}}{R}, \\
\left(\frac{f \circ \pi_{1} \circ \pi_{5}}{\lambda_{4}}\right) \circ \pi_{4} & \leq \frac{\|f\|_{\tilde{\rho}, \xi}}{R} .
\end{aligned}
$$

When estimating $a_{3}$ and $a_{4}$ in case (30) we also use the inequality

$$
\left|\lambda_{4}\right| \leq\left|\xi_{4}\right|+1000 \sqrt{R}<1+\frac{1}{2}=\frac{3}{2} .
$$

It is now easy to check that (26) holds.

6.3. Growth constant for the Bautin ideal $I_{\rho}(\xi)$ relative to canonical generators. In Subsection 6.2, we found a growth constant for the Bautin ideal $I_{\rho}(\xi)$ relative to the Dulac generators $f_{1}, \ldots, f_{4}$ in the marked polydisk $\Delta_{\tilde{\rho}}(\xi)$ centered at $\xi \in \Sigma$. Now we want to give an estimate on the growth constant for that ideal relative to the canonical generators $g_{1}, g_{3}, g_{5}, g_{7}$. First, we need a preliminary result on analytic continuation of the Poincaré map $P_{\lambda}(x)$ along $x$ in a neighborhood of the origin.

Proposition 7. Suppose $\left|\lambda_{1}\right| \leq \frac{1}{2}$ and $\left|\lambda_{i}\right| \leq 2$ for $i=2, \ldots, 6$. Then $P_{\lambda}(x)$ is defined for $x \in \mathbb{C}$ in the disk $|x| \leq 10^{-8}$ with its modulus bounded there by $\frac{1}{40}$.

Proof. Set $\rho=z \bar{z}$. It follows from (17) that

$$
\frac{d \rho}{d \theta}=2 \rho \operatorname{Re}\left(\frac{\lambda_{1}+f_{\lambda}(\theta) z}{1+g_{\lambda}(\theta) z}\right)
$$

Inequalities (17) imply that, for the imposed bounds on $\left|\lambda_{i}\right|$, we have the inequalities

$$
\begin{aligned}
& \left|f_{\lambda}(\theta)\right| \leq 20, \\
& \left|g_{\lambda}(\theta)\right| \leq 20,
\end{aligned}
$$

which, for $|z| \leq \frac{1}{40}$ and $\theta \in[0,2 \pi]$, imply that

$$
\left|\operatorname{Re}\left(\frac{\lambda_{1}+f_{\lambda}(\theta) z}{1+g_{\lambda}(\theta) z}\right)\right| \leq 2\left|\lambda_{1}\right|+1 .
$$

Thus if, for $\theta \in\left[0, \theta_{0}\right]$, the solution of (17) satisfies the condition $|z(\lambda, \theta, x)| \leq \frac{1}{40}$, then for those $\theta$ we have

$$
\frac{d \rho}{d \theta} \leq 2 \rho\left(2\left|\lambda_{1}\right|+1\right)
$$


Integrating this inequality with respect to $\theta$ from 0 to $\theta_{0}$, we have

$$
\ln \left(\frac{\rho\left(\lambda, \theta_{0}\right)}{\rho(\lambda, 0)}\right) \leq 2 \theta_{0}\left(2\left|\lambda_{1}\right|+1\right),
$$

i.e., $\rho\left(\lambda, \theta_{0}\right) \leq \rho(\lambda, 0) \exp \left(2 \theta_{0}\left(2\left|\lambda_{1}\right|+1\right)\right)$. As $|z|^{2}=\rho$, we then have

$$
\left|z\left(\lambda, \theta_{0}, x\right)\right| \leq|z(\lambda, 0, x)| \exp \left(\theta_{0}\left(2\left|\lambda_{1}\right|+1\right)\right)=|x| \exp \left(\theta_{0}\left(2\left|\lambda_{1}\right|+1\right)\right) .
$$

Suppose

$$
|x| \leq 10^{-8} \leq \frac{\exp \left(-2 \pi\left(2\left|\lambda_{1}\right|+1\right)\right)}{40}
$$

(the last inequality is true because $\left.\left|\lambda_{1}\right| \leq 1 / 2\right)$. Taking into account the inequality $\left|\theta_{0}\right| \leq$ $2 \pi$ and estimate (32), we have $\left|z\left(\lambda, \theta_{0}, x\right)\right| \leq \frac{1}{40}$. Therefore, $|z(\lambda, 2 \pi, x)|=\left|P_{\lambda}(x)\right| \leq \frac{1}{40}$, as claimed.

Recall that

$$
\left(\begin{array}{l}
g_{1} \\
g_{3} \\
g_{5} \\
g_{7}
\end{array}\right)=\left(\begin{array}{cccc}
a_{11} & 0 & 0 & 0 \\
a_{21} & a_{22} & 0 & 0 \\
a_{31} & a_{32} & a_{33} & 0 \\
a_{41} & a_{42} & a_{43} & a_{44}
\end{array}\right)\left(\begin{array}{l}
f_{1} \\
f_{2} \\
f_{3} \\
f_{4}
\end{array}\right)=A \cdot\left(\begin{array}{c}
c f_{1} \\
f_{2} \\
f_{3} \\
f_{4}
\end{array}\right)
$$

where $A$ is a holomorphic matrix. To find a growth constant relative to the canonical generators we need to express $f_{1}, \ldots, f_{4}$ in terms of $g_{1}, g_{3}, g_{5}, g_{7}$ and find an upper bound on the coefficients in that decomposition. To that end, we shall find an upper bound on the nondiagonal coefficients of $A$ and determine the diagonal coefficients.

In general, the coefficients $a_{i j}$ are not unique. Recall that $f_{1}=\lambda_{1}$. We shall assume that the $a_{i j}$ satisfy the following condition: all $a_{i j}$ for $j \neq 1$ do not depend on $\lambda_{1}$, i.e., $a_{i j}\left(\lambda_{1}, \bar{\lambda}\right)=a_{i j}(0, \bar{\lambda})$. Indeed, in Lemma 6 we explicitly found the coefficients in the decomposition by the Dulac generators for any function in the Bautin ideal. It is not difficult to see that they are all, except the coefficient of $f_{1}$, not dependent on $\lambda_{1}$. Now our plan is as follows.

1) First, we set $\lambda_{1}=0$ and determine all entries $a_{i j}$ of $A$ for $j \neq 1$ (i.e., all entries but those in the first column). The diagonal entries of $A$ will be constant, and the nondiagonal ones will be estimated from above;

2) Then, for $\lambda_{1} \neq 0$ we shall find an explicit expression for $a_{11}$ and estimate it from below. The remaining coefficients of the first column of $A$ will be estimated from above. For that, we shall use Proposition 7 and apply the Cauchy estimates to find estimates on the moduli of the Taylor series coefficients for $D_{\lambda}(x)$ (i.e., $\left|g_{i}(\lambda)\right|$ for $\left.i \in\{3,5,7\}\right)$. Also, using

$$
a_{i 1}=\frac{g_{i}(\lambda)-\sum_{j>1} a_{i j} f_{j}}{\lambda_{1}},
$$

we shall estimate $\left|a_{i 1}\right|$. For that we use the Schwarz lemma and the already known estimates on $a_{i 2}, a_{i 3}, a_{i 4}$.

We now give the details.

Canonical generators of the Bautin ideal for $\lambda_{1}=0$. We want to find canonical generators $g_{i}$ of the Bautin ideal of the displacement function $D_{\lambda}(x)$ of the Poincaré map for $\lambda_{1}=0$. Suppose $z(\lambda, \theta, x)$ is the solution of (17) with initial value condition $z(\lambda, 0, x)=x$. It follows from the proof of Proposition 7 that $z(\lambda, \theta, x)$ is analytic in $x$ 
in a neighborhood of zero for any $\theta \in[0,2 \pi]$ when $\left|\lambda_{1}\right| \leq 1 / 2$ and $\left|\lambda_{i}\right| \leq 2(i=2, \ldots, 6)$. Expanding this function into a Taylor series in $x$ we have

$$
z(\lambda, \theta, x)=\sum_{i=1}^{\infty} v_{i}(\lambda, \theta) x^{i}
$$

The constant term of this series vanishes because $z(\lambda, \theta, 0) \equiv 0$. Equation (17) with $\lambda_{1}=0$ then becomes

$$
\frac{d z(\lambda, \theta, x)}{d \theta}=\sum_{i=2}^{\infty} R_{i}(\lambda, \theta) z^{i}
$$

where $R_{i}(\lambda, \theta)=(-1)^{i} f_{\lambda}(\theta) g_{\lambda}^{i-2}(\theta)$.

Lemma 8. Suppose $\lambda_{1}=0$. Consider the system of differential equations

$$
\begin{aligned}
\frac{d v_{1}}{d \theta}= & 0 \\
\frac{d v_{2}}{d \theta}= & v_{1}^{2} R_{2} \\
\frac{d v_{3}}{d \theta}= & 2 v_{1} v_{2} R_{2}+v_{1}^{3} R_{3} \\
\frac{d v_{4}}{d \theta}= & \left(2 v_{1} v_{3}+v_{2}^{2}\right) R_{2}+3 v_{1}^{2} v_{2} R_{3}+v_{1}^{4} R_{4} \\
\frac{d v_{5}}{d \theta}= & 2\left(v_{1} v_{4}+v_{2} v_{3}\right) R_{2}+3 v_{1}\left(v_{1} v_{3}+v_{2}^{2}\right) R_{3}+4 v_{1}^{3} v_{2} R_{4}+v_{1}^{5} R_{5} \\
\frac{d v_{6}}{d \theta}= & \left(2 v_{1} v_{5}+2 v_{2} v_{4}+v_{3}^{2}\right) R_{2}+\left(3 v_{1}^{2} v_{4}+6 v_{1} v_{2} v_{3}+v_{2}^{3}\right) R_{3} \\
& +2 v_{1}^{2}\left(2 v_{1} v_{3}+3 v_{2}^{2}\right) R_{4}+5 v_{1}^{4} v_{2} R_{5}+v_{1}^{6} R_{6}, \\
\frac{d v_{7}}{d \theta}= & 2\left(v_{1} v_{6}+v_{2} v_{5}+v_{3} v_{4}\right) R_{2}+3\left(v_{1}^{2} v_{5}+2 v_{1} v_{2} v_{4}+v_{1} v_{3}^{2}+v_{2}^{2} v_{3}\right) R_{3} \\
& +4 v_{1}\left(v_{1}^{2} v_{4}+3 v_{1} v_{2} v_{3}+v_{2}^{3}\right) R_{4}+5 v_{1}^{3}\left(2 v_{2}^{2}+v_{1} v_{3}\right) R_{5}+6 v_{1}^{5} v_{2} R_{6}+v_{1}^{7} R_{7} .
\end{aligned}
$$

Set the initial values for $\theta=0$ :

$$
v_{1}(\lambda, 0)=1, \quad v_{2}(\lambda, 0)=\cdots=v_{7}(\lambda, 0)=0 .
$$

The canonical generators $g_{i}(\lambda)$ of the Bautin ideal of $D_{\lambda}(x)$ at $x=0$ and the solutions $v_{i}(\lambda, \theta)$ of (35) with initial values (36) are related as follows:

$$
g_{1}(\lambda)=v_{1}(\lambda, 2 \pi)-1=0 ; \quad g_{i}(\lambda)=v_{i}(\lambda, 2 \pi) \quad \text { for } i>1 .
$$

Proof. Substituting series (33) into (34) and comparing the coefficients of the powers of $x$, we have equations (35). The initial conditions $z(\lambda, 0, x)=x$ then transform into the initial conditions (36). As

$$
D_{\lambda}(x)=P_{\lambda}(x)-x=\left(v_{1}(\lambda, 2 \pi)-1\right) x+\sum_{i=2}^{\infty} v_{i}(\lambda, 2 \pi) x^{i},
$$

relations (37) now follow from the definition of the canonical generators $g_{i}(\lambda)$.

The coefficients $a_{i j}$ with $j \neq 1$. System (35) with initial conditions (36) is easy to solve. Indeed, $v_{1} \equiv 1$, and for the known $v_{1}, \ldots, v_{i}$, the right-hand side of the equation for $d v_{i+1} / d \theta$ becomes a known polynomial in $\lambda_{2}, \ldots, \lambda_{6}, \cos \theta, \sin \theta$. Integrating this function and substituting the initial condition (36), we determine $v_{i+1}$. Taking $\theta=2 \pi$, we obtain $g_{i}(\lambda)=g_{i}\left(0, \lambda_{2}, \ldots, \lambda_{6}\right)$ as polynomials in $\lambda_{2}, \ldots, \lambda_{6}$. For these calculations 
we used the computer algebra system Mathematica. The corresponding program can be found in the Appendix.

Lemma 9. We have the following equalities:

$$
\begin{aligned}
& g_{3}\left(0, \lambda_{2}, \ldots, \lambda_{6}\right)=a_{22} f_{2}, \\
& g_{5}\left(0, \lambda_{2}, \ldots, \lambda_{6}\right)=a_{32} f_{2}+a_{33} f_{3}, \\
& g_{7}\left(0, \lambda_{2}, \ldots, \lambda_{6}\right)=a_{42} f_{2}+a_{43} f_{3}+a_{44} f_{4},
\end{aligned}
$$

where

(39)

$$
\begin{aligned}
& a_{22}=-\pi / 4 \\
& a_{32}=-\frac{1}{288}\left(168 \pi \lambda_{2}^{2}+90 \pi \lambda_{3}^{2}+21 \pi \lambda_{3} \lambda_{4}+\pi \lambda_{4}^{2}+108 \pi \lambda_{2} \lambda_{5}\right. \\
& \left.-27 \pi^{2} \lambda_{3} \lambda_{5}+21 \pi \lambda_{5}^{2}+35 \pi \lambda_{4} \lambda_{6}+27 \pi^{2} \lambda_{5} \lambda_{6}+70 \pi \lambda_{6}^{2}\right), \\
& a_{33}=\pi / 24 \text {, } \\
& a_{42}=-\frac{1}{276480}\left(417280 \pi \lambda_{2}^{4}+633600 \pi \lambda_{2}^{2} \lambda_{3}^{2}+170100 \pi \lambda_{3}^{4}-81216 \pi \lambda_{2}^{2} \lambda_{3} \lambda_{4}\right. \\
& +57600 \pi^{2} \lambda_{2} \lambda_{3}^{2} \lambda_{4}+82260 \pi \lambda_{3}^{3} \lambda_{4}-31200 \pi \lambda_{2}^{2} \lambda_{4}^{2} \\
& +11520 \pi^{2} \lambda_{2} \lambda_{3} \lambda_{4}^{2}+12945 \pi \lambda_{3}^{2} \lambda_{4}^{2}+690 \pi \lambda_{3} \lambda_{4}^{3}+5 \pi \lambda_{4}^{4} \\
& +624832 \pi \lambda_{2}^{3} \lambda_{5}-165120 \pi^{2} \lambda_{2}^{2} \lambda_{3} \lambda_{5}+432288 \pi \lambda_{2} \lambda_{3}^{2} \lambda_{5} \\
& -86400 \pi^{2} \lambda_{3}^{3} \lambda_{5}+36096 \pi \lambda_{2} \lambda_{3} \lambda_{4} \lambda_{5}-20160 \pi^{2} \lambda_{3}^{2} \lambda_{4} \lambda_{5} \\
& -6720 \pi \lambda_{2} \lambda_{4}^{2} \lambda_{5}-960 \pi^{2} \lambda_{3} \lambda_{4}^{2} \lambda_{5} \\
& +382272 \pi \lambda_{2}^{2} \lambda_{5}^{2}-111360 \pi^{2} \lambda_{2} \lambda_{3} \lambda_{5}^{2} \\
& +\left(87003 \pi+10800 \pi^{3}\right) \lambda_{3}^{2} \lambda_{5}^{2}+19722 \pi \lambda_{3} \lambda_{4} \lambda_{5}^{2}+930 \pi \lambda_{4}^{2} \lambda_{5}^{2} \\
& +105208 \pi \lambda_{2} \lambda_{5}^{3}-24000 \pi^{2} \lambda_{3} \lambda_{5}^{3}+10813 \pi \lambda_{5}^{4}-403200 \pi \lambda_{2}^{2} \lambda_{3} \lambda_{6} \\
& -50400 \pi \lambda_{3}^{3} \lambda_{6}+347712 \pi \lambda_{2}^{2} \lambda_{4} \lambda_{6}-115200 \pi^{2} \lambda_{2} \lambda_{3} \lambda_{4} \lambda_{6} \\
& +60060 \pi \lambda_{3}^{2} \lambda_{4} \lambda_{6}-11520 \pi^{2} \lambda_{2} \lambda_{4}^{2} \lambda_{6}+10710 \pi \lambda_{3} \lambda_{4}^{2} \lambda_{6}+350 \pi \lambda_{4}^{3} \lambda_{6} \\
& +165120 \pi^{2} \lambda_{2}^{2} \lambda_{5} \lambda_{6}-179616 \pi \lambda_{2} \lambda_{3} \lambda_{5} \lambda_{6}+86400 \pi^{2} \lambda_{3}^{2} \lambda_{5} \lambda_{6} \\
& +169056 \pi \lambda_{2} \lambda_{4} \lambda_{5} \lambda_{6}-13440 \pi^{2} \lambda_{3} \lambda_{4} \lambda_{5} \lambda_{6}+960 \pi^{2} \lambda_{4}^{2} \lambda_{5} \lambda_{6} \\
& +111360 \pi^{2} \lambda_{2} \lambda_{5}^{2} \lambda_{6}+\left(-4686 \pi-21600 \pi^{3}\right) \lambda_{3} \lambda_{5}^{2} \lambda_{6}+32934 \pi \\
& +32934 \pi \lambda_{4} \lambda_{5}^{2} \lambda_{6} \lambda_{4} \lambda_{5}^{2} \lambda_{6}+24000 \pi^{2} \lambda_{5}^{3} \lambda_{6}+537600 \pi \lambda_{2}^{2} \lambda_{6}^{2} \\
& +138600 \pi \lambda_{3}^{2} \lambda_{6}^{2}+57600 \pi^{2} \lambda_{2} \lambda_{4} \lambda_{6}^{2}+4620 \pi \lambda_{3} \lambda_{4} \lambda_{6}^{2} \\
& +21945 \pi \lambda_{4}^{2} \lambda_{6}^{2}+344256 \pi \lambda_{2} \lambda_{5} \lambda_{6}^{2}-67200 \pi^{2} \lambda_{3} \lambda_{5} \lambda_{6}^{2} 2 \\
& +33600 \pi^{2} \lambda_{4} \lambda_{5} \lambda_{6}^{2}+\left(68211 \pi+10800 \pi^{3}\right) \lambda_{5}^{2} \lambda_{6}^{2} \\
& \left.+100100 \pi \lambda_{4} \lambda_{6}^{3}+67200 \pi^{2} \lambda_{5} \lambda_{6}^{3}+100100 \pi \lambda_{6}^{4}\right), \\
& a_{43}=\frac{\pi}{276480}\left(48960 \lambda_{2}^{2}+45360 \lambda_{3}^{2}+16920 \lambda_{3} \lambda_{4}\right. \\
& \left.+1560 \lambda_{4}^{2}+1440 \lambda_{3} \lambda_{6}+2760 \lambda_{4} \lambda_{6}+1200 \lambda_{6}^{2}\right), \\
& a_{44}=-\frac{5 \pi}{32} .
\end{aligned}
$$


For $\left|\lambda_{i}\right| \leq 2$, we also have:

$$
\begin{aligned}
& \left|a_{32}\right| \leq 37, \\
& \left|a_{42}\right| \leq 2114, \\
& \left|a_{43}\right| \leq 7 .
\end{aligned}
$$

Proof. Coefficients (39) were obtained with the aid of the computer program described in the Appendix. Inequalities (40) follow from (39) if one estimates the modulus of each summand using the fact that $\left|\lambda_{i}\right| \leq 2$, and then adds the obtained estimates.

Estimates on the coefficients $a_{i 1}$. Now we want to estimate the entries in the first column of $A$. Unlike the other entries of the matrix, they, in general, depend on $\lambda_{1}$. Because of that, we shall henceforth assume that $\lambda_{1}$ is an arbitrary complex number such that $\left|\lambda_{1}\right| \leq \frac{1}{2}$.

Lemma 10. Suppose that $\left|\lambda_{1}\right| \leq \frac{1}{2}$ and $\left|\lambda_{i}\right| \leq 2$ for $i=2, \ldots, 6$. Then:

$$
\begin{aligned}
& \left|a_{11}(\lambda)\right| \geq 1, \\
& \left|a_{21}(\lambda)\right| \leq 10^{23}, \\
& \left|a_{31}(\lambda)\right| \leq 10^{39} \\
& \left|a_{41}(\lambda)\right| \leq 10^{55} .
\end{aligned}
$$

Proof. It follows from the variational equation for the derivative of the solution of (17) with respect to initial conditions that

$$
g_{1}(\lambda)=e^{2 \pi \lambda_{1}}-1=\lambda_{1} \frac{e^{2 \pi \lambda_{1}}-1}{\lambda_{1}}=f_{1} \frac{e^{2 \pi \lambda_{1}}-1}{\lambda_{1}} .
$$

Thus,

$$
a_{11}=\frac{e^{2 \pi \lambda_{1}}-1}{\lambda_{1}} .
$$

This function is holomorphic and does not vanish; hence its minimum on the disk $\left|\lambda_{1}\right| \leq \frac{1}{2}$ is attained on the circle $\left|\lambda_{1}\right|=\frac{1}{2}$. It is now clear that, for $\left|\lambda_{1}\right| \leq \frac{1}{2}$,

$$
\left|a_{11}\right| \geq \frac{1-e^{-\pi}}{1 / 2}>1 \text {. }
$$

Now we want to find an estimate on $\left|a_{21}(\lambda)\right|,\left|a_{31}(\lambda)\right|$, and $\left|a_{41}(\lambda)\right|$ from above. Since $\left|\lambda_{1}\right| \leq 1 / 2$ and $\left|\lambda_{i}\right| \leq 2$, we apply Proposition 7 and find the Cauchy estimates for the coefficients $g_{k}(\lambda)$ of the Taylor series for $P_{\lambda}(x)$ with $k>1$ :

$$
\left|g_{k}(\lambda)\right| \leq \frac{1 / 40}{10^{-8 k}} \text {. }
$$

It follows from formulas (4) and (5) for the Dulac generators that, for $\left|\lambda_{i}\right| \leq 2$,

$$
\begin{aligned}
& \left|f_{2}\right| \leq 8, \\
& \left|f_{3}\right| \leq 352, \\
& \left|f_{4}\right| \leq 1024 .
\end{aligned}
$$

Since $\left|a_{22}\right|=\frac{\pi}{4}$ and

$$
a_{21}(\lambda)=\frac{g_{3}(\lambda)-a_{22}(\lambda) f_{2}(\lambda)}{\lambda_{1}},
$$

the Schwarz lemma, applied in the disk $\left|\lambda_{1}\right| \leq \frac{1}{2}$, shows that

$$
\left|a_{21}\right| \leq 2\left(\frac{10^{24}}{40}+\frac{\pi}{4} \cdot 8\right) \leq 10^{23} \text {. }
$$


Similarly, the equality

$$
a_{31}=\frac{g_{5}-a_{32} f_{2}-a_{33} f_{3}}{\lambda_{1}},
$$

together with (39), (40), (42), (43), and the Schwarz lemma, shows that

$$
\left|a_{31}\right| \leq 2\left(\frac{10^{40}}{40}+\frac{\pi}{24} \cdot 352+37 \cdot 8\right) \leq 10^{39} .
$$

Finally, the equality

$$
a_{41}=\frac{g_{7}-a_{42} f_{2}-a_{43} f_{3}-a_{44} f_{4}}{\lambda_{1}}
$$

implies that, in the disk $\left|\lambda_{1}\right| \leq \frac{1}{2}$,

$$
\left|a_{41}\right| \leq 2\left(\frac{10^{56}}{40}+2114 \cdot 8+7 \cdot 352+\frac{5 \pi}{32} \cdot 1024\right) \leq 10^{55} .
$$

The desired estimates have been obtained.

Recalculation of the growth constant for the canonical generators. We now have all the tools to estimate the growth constant for the Bautin ideal with canonical generators in a sufficiently small marked polydisk.

Lemma 11. Suppose $\xi \in \Sigma, 0<R<10^{-7}$, and $\Delta_{\rho}(\xi)$ is a marked $R$-polydisk. Suppose also that $f \in O\left(\Delta_{\rho}(\xi)\right)$ and the germ of $f$ on $\Sigma$ belongs to the Bautin ideal $I(\Sigma)$. Then there are functions $b_{1}, b_{3}, b_{5}, b_{7} \in O\left(\overline{\Delta_{\rho}(\xi)}\right)$ such that

$$
f=b_{1} g_{1}+b_{3} g_{3}+b_{5} g_{5}+b_{7} g_{7}
$$

and, for some $\varepsilon>0$,

$$
\left\|b_{i}\right\|_{\tilde{\rho}, \xi} \leq D\|f\|_{\tilde{\rho}, \xi}, \text { where } D=\frac{10^{56}}{R^{7}},
$$

in any polydisk $\Delta_{\tilde{\rho}}(\xi) \subset \Delta_{\rho}(\xi)$, provided $\|\tilde{\rho}-\rho\|<\varepsilon$.

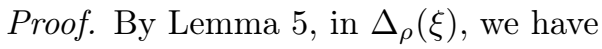

$$
f=a_{1} f_{1}+a_{2} f_{2}+a_{3} f_{3}+a_{4} f_{4}
$$

and

$$
\left\|a_{i}\right\|_{\tilde{\rho}, \xi} \leq C\|f\|_{\tilde{\rho}, \xi}, \text { where } C=\frac{3}{R^{7}},
$$

for any polyradius $\tilde{\rho}<\rho$ sufficiently close to $\rho$. Using the transition matrix $A$, we express $f_{1}, \ldots, f_{4}$ in terms of $g_{1}, g_{3}, g_{5}, g_{7}$, and regroup the summands in (46) to find the coefficients $b_{1}, b_{3}, b_{5}, b_{7}$ in (44). We then have

$$
\begin{aligned}
b_{1}= & \left(\frac{a_{42} a_{21}}{a_{11} a_{22} a_{44}}+\frac{a_{43} a_{31}}{a_{11} a_{33} a_{44}}+\frac{a_{43} a_{32} a_{21}}{a_{11} a_{22} a_{33} a_{44}}-\frac{a_{41}}{a_{11} a_{44}}\right) a_{4} \\
& -\left(\frac{a_{31}}{a_{11} a_{33}}+\frac{a_{32} a_{21}}{a_{11} a_{22} a_{33}}\right) a_{3}-\frac{a_{21}}{a_{11} a_{22}} a_{2}+\frac{1}{a_{11}} a_{1}, \\
b_{3}= & \left(\frac{a_{43} a_{32}}{a_{44} a_{22} a_{33}}-\frac{a_{42}}{a_{44} a_{22}}\right) a_{4}-\frac{a_{32}}{a_{22} a_{33}} a_{3}+\frac{1}{a_{22}} a_{2}, \\
b_{5}= & \frac{1}{a_{33}} a_{3}-\frac{a_{43}}{a_{44} a_{33}} a_{4}, \\
b_{7}= & \frac{1}{a_{44}} a_{4} .
\end{aligned}
$$


Since $R<10^{-7}$, Lemma 4 shows that the components $\rho_{i}$ of $\rho$ satisfy the inequality

$$
\rho_{i} \leq 1000 \sqrt{R}<\frac{1}{2}
$$

Since $\xi_{1}=0$ and $\left|\xi_{i}\right| \leq 1$, we have $\left|\lambda_{1}\right| \leq \frac{1}{2}$ and $\left|\lambda_{i}\right| \leq 2$, for $i=2, \ldots, 6$, in $\Delta_{\rho}(\xi)$. Therefore, for $\lambda \in \Delta_{\tilde{\rho}}(\xi)$, we can apply the estimates on the coefficients $a_{i j}$ from Lemmas 9 and 10. Using them to estimate the right-hand sides of (48) and taking account of (47), we have (45). Lemma 11 is proved.

Lemma 3 now follows at once from the just proved lemma. To see this, consider a marked $R$-polydisk $\Delta_{\tilde{\rho}}(\xi)$ with $R<10^{-7}$. If $f \in O\left(\overline{\Delta_{\tilde{\rho}}(\xi)}\right) \cap I(\Sigma)$, then we can choose a polyradius $\rho>\tilde{\rho}$ such that the polydisk $\Delta_{\rho}(\xi)$ is again a suitable $R$-polydisk, $f$ is holomorphic on it, and Lemma 11 holds for the polyradii $\rho, \tilde{\rho}$. Thus, the polydisk $\Delta_{\tilde{\rho}}(\xi)$ is suitable for the Bautin ideal $I_{\tilde{\rho}}(\xi)$, and estimates (45) hold on it. The components $\tilde{\rho}_{i}$ of the polyradius of that polydisk are such that

$$
R<\tilde{\rho}_{i}<1000 \sqrt{R}
$$

which finishes the proof of Lemma 3

\section{Complex normal Form And the Ilyashenko-Llibre theorem}

In this section we state the Ilyashenko-Llibre theorem ([5]) providing an estimate on the number of $\delta$-good limit cycles for quadratic vector fields which are $\kappa$-distant from singular fields and $\sigma$-distant from centers (see Theorem 5 below). The corresponding statement in [5] is formulated for vector fields in complex normal form. In that case, the concepts of $\delta$-good limit cycles and vector fields $\sigma$-close to centers and $\kappa$-distant from singular fields also differ from the ones considered above. First, we need some definitions.

Complex normal form. Consider the quadratic vector field (11) with focus-type singular point at the origin. Using a linear change of coordinates and time, we rewrite it as

$$
\left\{\begin{array}{l}
\dot{x}=\lambda_{1} x-y+\cdots \\
\dot{y}=x+\lambda_{1} y+\cdots
\end{array}\right.
$$

where the ellipses indicate quadratic monomials. Introducing the complex variables

$$
z=x+i y, \quad \bar{z}=x-i y,
$$

we rewrite the system as one complex equation whose real and imaginary parts correspond to the previous equations for $\dot{x}$ and $\dot{y}$ :

$$
\dot{z}=\mu z+A z^{2}+B z \bar{z}+C \bar{z}^{2} .
$$

Here, the coefficients $A, B$, and $C$ take on complex values and $\mu=\lambda_{1}+i$. The substitution $z \rightarrow c z$, where $c \in \mathbb{C}$, transforms this equation to

$$
\dot{z}=\mu z+A c z^{2}+B \bar{c} z \bar{z}+C \frac{\bar{c}^{2}}{c} \bar{z}^{2}
$$

and allows us to normalize the coefficients of (49) so as to satisfy one of the following three conditions:

$$
\begin{array}{ll}
A=1, & |B| \leq 2, \quad|C| \leq 1 \\
|A| \leq 1, & B=2, \quad|C| \leq 1 \\
|A| \leq 1, & |B| \leq 2, \quad C=1
\end{array}
$$


Definition 10. Equation (49) with coefficients satisfying one of the conditions (51)-(53) is called a quadratic equation in complex normal form. Henceforth, when speaking of the vector field (49), we shall mean the vector field in $\mathbb{R}^{2}$ given by this equation.

Suppose $\delta>0$. Consider the disk of radius $\frac{1}{\delta}$ centered at the origin in the phase plane $\mathbb{R}^{2}=(\operatorname{Re} z, \operatorname{Im} z)$. In this disk, we remove the $\delta$-neighborhoods of all singular points of vector field (49) (including complex neighborhoods of complex singular points in $\mathbb{C}^{2}$ ), except for the origin. Denote this set by $D(\mu, A, B, C, \delta)$. The following definition is completely analogous to the definition of a $\delta$-good limit cycle from Section 1

Definition 11. A limit cycle of the vector field (49) in complex normal form is said to be $\delta$-good if it encircles the origin and is contained in $D(\mu, A, B, C, \delta)$.

Definitions for vector fields, $\sigma$-close to centers and $\kappa$-distant from singular fields, in complex normal form, differ from the corresponding Definitions 3 and 4 for vector fields in Kapteyn's normal form.

Conditions for centers in complex normal form and vector fields close to centers. Define the polynomials

$$
\begin{aligned}
& h_{1}:=\lambda_{1}, \\
& h_{2}:=\operatorname{Im}(A B), \\
& h_{3}:=\operatorname{Im}[(2 A+\bar{B})(A-2 \bar{B}) \bar{B} C], \\
& h_{4}:=\operatorname{Im}\left[(2 A+\bar{B})\left(|B|^{2}-|C|^{2}\right) \bar{B}^{2} C\right] .
\end{aligned}
$$

The simultaneous vanishing of these polynomials is equivalent to the singular point of the vector field (49) at the origin being a center ([14], [7]).

Definition 12. The ideal generated by the polynomials $h_{1}, \ldots, h_{4}$ is called the Dulac ideal for equation (49) in complex normal form, and its generators $h_{i}$ are called Żołacdek generators.

Definition 13. The vector field (49) in complex normal form for given values of the parameters $\lambda_{1}, A, B, C$ is said to be $\sigma$-close to centers if

$$
\sum_{j=1}^{4}\left|h_{j}\right|<\sigma .
$$

Otherwise, it is said to be $\sigma$-distant from centers.

Vector fields which are far from singular fields in complex normal form. In [5], it was shown that a singular vector field in complex normal form is of the form

$$
\dot{z}=\mu z+z^{2}+\frac{\mu}{\bar{\mu}} z \bar{z} ;
$$

i.e., it corresponds to (51) with $A=1, B=\frac{\mu}{\bar{\mu}}$, and $C=0$. It is not difficult to see that under a small perturbation of the parameters of a singular vector field, the complex normal form again corresponds to (51). This motivates the following definition.

Definition 14. The vector field (49) in the complex normal form corresponding to (51) is said to be $\kappa$-distant from singular fields if

$$
\sqrt{\left|B-\frac{\mu}{\bar{\mu}}\right|^{2}+|C|^{2}}>\kappa .
$$

Notice that this definition looks more natural than Definition 4 for Kapteyn's normal form. Nevertheless, in Subsection 8.1, we shall show that the relations between the parameters $\kappa$ for different normal forms are very simple. 
The Ilyashenko-Llibre theorem. We have given all the necessary definitions to be able to state the Ilyashenko-Llibre theorem [5].

Theorem 5. Suppose that $\{\delta, \sigma, \kappa\} \subset(0,0.1)$ and that the vector field (49) in complex normal form is $\sigma$-distant from centers and $\kappa$-distant from singular fields (in the sense of Definitions 13 and 14). Then the number of $\delta$-good limit cycles of that vector field is at most

$$
|\ln \sigma| \exp \left[\exp \left(10^{25} \delta^{-31} \kappa^{-2}\right)\right] .
$$

\section{Proof of Proposition 2 and Theorem 2}

In this section, we shall prove Proposition 2 and Theorem 2 The main difficulty we are facing comes from the use of different normal forms in [5] and in this paper. This is related to the fact that even though the complex normal form is simpler, Kapteyn's normal form is more convenient for estimating the growth constant of the Bautin ideal and for the proof of Lemma 3

To prove Proposition 2 and Theorem 2, we need to relate the parameters $\delta, \sigma$, and $\kappa$ in different normal forms. First, we shall switch from system $\left(2_{\lambda}\right)$ in Kapteyn's normal form to the complex normal form and obtain some auxiliary estimates. The substitution $z=x+i y$ transforms system $\left(2_{\lambda}\right)$ into

$$
\dot{z}=\mu z+A_{0} z^{2}+B_{0} z \bar{z}+C_{0} \bar{z}^{2},
$$

where

$$
\mu=\lambda_{1}+i, \quad A_{0}=\frac{1}{4} L_{1}+\frac{1}{4} L_{2}, \quad B_{0}=-\frac{1}{2} L_{1}, \quad C_{0}=\frac{1}{4} L_{1}-\frac{1}{4} L_{2}-L_{3}
$$

and

$$
L_{1}=\lambda_{3}-\lambda_{6}, \quad L_{2}=\lambda_{4}+i \lambda_{5}, \quad L_{3}=\lambda_{3}-i \lambda_{2} .
$$

To obtain the complex normal form, it remains to normalize the coefficients in equation (55) using a suitable substitution $z \rightarrow c z$. For $\lambda \in \Lambda$, we shall find estimates on $|c|$ from above and below.

Proposition 8. For $\lambda \in \Lambda$ we have:

$$
\left|A_{0}\right| \leq 1, \quad\left|B_{0}\right| \leq 1, \quad\left|C_{0}\right| \leq 3,
$$

and either $\left|A_{0}\right|>\frac{1}{18}$ or $\left|B_{0}\right|>\frac{1}{18}$ or $\left|C_{0}\right|>\frac{1}{9}$.

Proof. When $\lambda \in \Lambda$, we have $\left|\lambda_{j}\right| \leq 1(j=2, \ldots, 6)$, whence (57). Suppose now that the following inequalities hold simultaneously:

$$
\left|A_{0}\right| \leq \frac{1}{18}, \quad\left|B_{0}\right| \leq \frac{1}{18}, \quad\left|C_{0}\right| \leq \frac{1}{9} .
$$

Then $\left|L_{1}\right|=2\left|B_{0}\right| \leq \frac{1}{9}$, hence $\left|L_{2}\right| \leq 4\left|A_{0}\right|+\left|L_{1}\right| \leq \frac{1}{3}$, and, by the definition of $L_{2}$, we have $\left|\lambda_{4}\right| \leq \frac{1}{3}$ and $\left|\lambda_{5}\right| \leq \frac{1}{3}$. Furthermore, $\left|L_{3}\right| \leq\left|C_{0}\right|+\frac{1}{4}\left|L_{1}\right|+\frac{1}{4}\left|L_{2}\right| \leq \frac{2}{9}$, whence $\left|\lambda_{3}\right| \leq \frac{2}{9}$ and $\left|\lambda_{2}\right| \leq \frac{2}{9}$. Finally, $\left|\lambda_{6}\right| \leq\left|L_{1}\right|+\left|\lambda_{3}\right| \leq \frac{1}{3}$. It is now easy to see that

$$
\sum_{j=2}^{6}\left|\lambda_{j}\right|^{2}<1 \text {, }
$$

contrary to the inclusion $\lambda \in \Lambda$. The proposition is proved.

Corollary 4. To bring equation (55) corresponding to $\lambda \in \Lambda$ to complex normal form, one has to use a substitution $z \rightarrow c z$, where $\frac{1}{3} \leq|c| \leq 36$.

Proof. This follows from (50), (51)-(53), and the estimates from Proposition 8 , 


\subsection{Relations between parameters $\delta, \sigma$, and $\kappa$ in different normal forms.}

In Propositions 9 11, proved below, we shall obtain estimates relating the parameters $\delta, \sigma$, and $\kappa$ in Kapteyn's normal form and in the complex normal form.

Proposition 9. Any $\delta$-good limit cycle of the vector field $\left[22_{\lambda}\right.$ in Kapteyn's normal form is a $\frac{\delta}{36}$-good limit cycle of that vector field in the complex normal form.

Proof. Consider a $\delta$-good limit cycle of the vector field $\left(2_{\lambda}\right)$ for $\lambda \in \Lambda$. To switch to complex normal form, we use a substitution $z \rightarrow c z$, which is a rotation in the phase plane about the origin and scaling with coefficient $\frac{1}{36} \leq\left|\frac{1}{c}\right| \leq 3$ (Corollary 4). Under such a transformation, a limit cycle of $\left(2_{\lambda}\right)$ contained in the disk of radius $\frac{1}{\delta}$ is mapped into the disk of radius $\frac{3}{\delta}$, and the distance from it to singular points cannot decrease more than 36 times. Therefore the cycle will be $\frac{\delta}{36}$-good in complex normal form.

Now we want to find a relation between the remoteness conditions for a vector field and singular fields in different normal forms. Recall that the vector field $\left(2_{\lambda}\right)$ is $\kappa$-distant from singular fields if $\left\|H_{\lambda}\right\|_{2}:=\sqrt{\int_{0}^{2 \pi} H_{\lambda}^{2}(\theta) d \theta}>\kappa$, where $\quad H_{\lambda}=\lambda_{1} g_{\lambda}-f_{\lambda}$, and $f_{\lambda}, g_{\lambda}$ are given by (7). It is not difficult to check that

$$
\begin{aligned}
\left\|H_{\lambda}\right\|_{2}^{2}= & 8\left(1+\lambda_{1}^{2}\right) \lambda_{2}^{2}+5 \lambda_{3}^{2}+4\left(1+\lambda_{1}^{2}\right) \lambda_{2} \lambda_{5}+\lambda_{5}^{2} \\
& +4 \lambda_{1} \lambda_{5}\left(-\lambda_{3}+\lambda_{6}\right)+\left(\lambda_{4}+\lambda_{6}\right)\left(2 \lambda_{3}+\lambda_{4}+\lambda_{6}\right) \\
& +\lambda_{1}^{2}\left(\left(3 \lambda_{3}+\lambda_{4}\right)^{2}+\lambda_{5}^{2}-2\left(3 \lambda_{3}+\lambda_{4}\right) \lambda_{6}+5 \lambda_{6}^{2}\right) .
\end{aligned}
$$

Proposition 10. Suppose $\delta<1$. If the vector field (2ג) in Kapteyn's normal form has $\delta$-good limit cycles and is $\kappa$-distant from singular fields, then in complex normal form it is $\kappa^{\prime}$-distant from singular fields, where $\kappa^{\prime}=\frac{\delta \kappa}{20}$.

Proof. Transforming $\left(2_{\lambda}\right)$ into complex normal form (49), (51), we have

$$
\mu=\lambda_{1}+i, \quad A=1, \quad B=\frac{B_{0}}{\overline{A_{0}}}, \quad C=\frac{C_{0} A_{0}}{{\overline{A_{0}}}^{2}},
$$

where $A_{0}, B_{0}, C_{0}$ are as in (56). Therefore

$$
\sqrt{\left|B-\frac{\mu}{\bar{\mu}}\right|^{2}+|C|^{2}}=\sqrt{\frac{\left|B_{0} \bar{\mu}-\overline{A_{0}} \mu\right|^{2}+\left|C_{0} \mu\right|^{2}}{\left|A_{0} \mu\right|^{2}}}=\frac{\sqrt{\left|B_{0} \bar{\mu}-\overline{A_{0}} \mu\right|^{2}+\left|C_{0} \mu\right|^{2}}}{\left|A_{0} \mu\right|} .
$$

By Proposition 1, we have $\left|\lambda_{1}\right| \leq \frac{10}{\delta}$, and therefore $|\mu| \leq 1+\left|\lambda_{1}\right|<\frac{11}{\delta}$. Since $\left|A_{0}\right| \leq 1$, the denominator $\left|A_{0} \mu\right|$ of the last fraction in (59) is at most $\frac{11}{\delta}$. The expression under the square root in the numerator is easy to compute. It is not difficult to see that

$$
\left|B_{0} \bar{\mu}-\overline{A_{0}} \mu\right|^{2}+\left|C_{0} \mu\right|^{2}=\frac{1}{\pi}\left\|H_{\lambda}\right\|_{2}^{2} .
$$

Thus, if $\left\|H_{\lambda}\right\|_{2}>\kappa$, then

$$
\sqrt{\left|B-\frac{\mu}{\bar{\mu}}\right|^{2}+|C|^{2}}>\frac{\delta \kappa}{20}:=\kappa^{\prime}
$$

and the vector field in complex normal form is $\kappa^{\prime}$-distant from singular fields.

Finally, we shall relate the conditions for remoteness from centers for a vector field in different normal forms.

Proposition 11. Consider the vector field (2ג) in Kapteyn's normal form. If, in complex normal form, it is $\sigma^{\prime}$-close to centers (in the sense of Definition 13) and $\sigma^{\prime} \leq 5^{-6}$, then it is $\sigma$-close to centers in the sense of Definition 3, where $\sigma=68 \sqrt[12]{\sigma^{\prime}}$. 
Proof. Transform $\left(2_{\lambda}\right)$ into complex normal form. Suppose $A_{0}, B_{0}, C_{0}$ are defined as in (56). Set

$$
\begin{aligned}
& h_{1}^{0}:=\lambda_{1}, \\
& h_{2}^{0}:=\operatorname{Im}\left(A_{0} B_{0}\right), \\
& h_{3}^{0}:=\operatorname{Im}\left[\left(2 A_{0}+\overline{B_{0}}\right)\left(A_{0}-2 \overline{B_{0}}\right) \overline{B_{0}} C_{0}\right], \\
& h_{4}^{0}:=\operatorname{Im}\left[\left(2 A_{0}+\overline{B_{0}}\right)\left(\left|B_{0}\right|^{2}-\left|C_{0}\right|^{2}\right){\overline{B_{0}}}^{2} C_{0}\right] .
\end{aligned}
$$

After normalization $z \rightarrow c z$, we have

$$
A=A_{0} c, \quad B=B_{0} \bar{c}, \quad C=C_{0} \frac{\bar{c}^{2}}{c},
$$

and therefore, since $|c|>1 / 3$,

$$
\left|h_{2}^{0}\right|=\frac{\left|h_{2}\right|}{|c|^{2}} \leq 9\left|h_{2}\right|, \quad\left|h_{3}^{0}\right|=\frac{\left|h_{3}\right|}{|c|^{4}} \leq 81\left|h_{3}\right|, \quad\left|h_{4}^{0}\right|=\frac{\left|h_{4}\right|}{|c|^{6}} \leq 729\left|h_{4}\right| .
$$

Suppose that the vector field in complex normal form is $\sigma^{\prime}$-close to centers, i.e.,

$$
\sum_{j=1}^{4}\left|h_{j}\right|<\sigma^{\prime}
$$

Then $\left|h_{j}\right|<\sigma^{\prime}$, and

$$
\left|h_{1}^{0}\right|<\sigma^{\prime}, \quad\left|h_{2}^{0}\right|<9 \sigma^{\prime}, \quad\left|h_{3}^{0}\right|<81 \sigma^{\prime}, \quad\left|h_{4}^{0}\right|<729 \sigma^{\prime} .
$$

To estimate the proximity of the original vector field $\left(2_{\lambda}\right)$ to centers, we estimate from above the Dulac generators $f_{1}, f_{2}, f_{3}, f_{4}$ given by (4) and (5). Clearly, $f_{1}=h_{1}^{0}=\lambda_{1}$, whence $\left|f_{1}\right|<\sigma^{\prime}$. To estimate the moduli of $f_{2}, f_{3}$, and $f_{4}$ we express these polynomials in terms of $h_{2}^{0}, h_{3}^{0}$, and $h_{4}^{0}$ :

$$
\left(\begin{array}{l}
f_{2} \\
f_{3} \\
f_{4}
\end{array}\right)=\left(\begin{array}{ccc}
b_{11} & 0 & 0 \\
b_{21} & b_{22} & 0 \\
b_{31} & b_{32} & b_{33}
\end{array}\right)\left(\begin{array}{l}
h_{2}^{0} \\
h_{3}^{0} \\
h_{4}^{0}
\end{array}\right)=B \cdot\left(\begin{array}{l}
h_{2}^{0} \\
h_{3}^{0} \\
h_{4}^{0}
\end{array}\right) .
$$

The entries $b_{i j}$ of the matrix $B$ can be computed using the computer algebra system Mathematica (see Appendix). The results are as follows:

$$
\begin{aligned}
& b_{11}=8, \\
& b_{21}=2\left(-15 \lambda_{3}^{2}-16 \lambda_{3} \lambda_{4}-3 \lambda_{4}^{2}+4 \lambda_{2} \lambda_{5}+\lambda_{5}^{2}+10 \lambda_{3} \lambda_{6}+8 \lambda_{4} \lambda_{6}+5 \lambda_{6}^{2}\right), \\
& b_{22}=-16, \\
& b_{31}= \frac{1}{8}\left(\lambda_{3}-\lambda_{6}\right)\left(-\lambda_{4}^{3}+3 \lambda_{4} \lambda_{5}^{2}-8 \lambda_{4}^{2} \lambda_{6}+8 \lambda_{5}^{2} \lambda_{6}+57 \lambda_{4} \lambda_{6}^{2}+32 \lambda_{6}^{3}\right. \\
& \quad+16 \lambda_{2}^{2}\left(3 \lambda_{3}+4 \lambda_{4}+\lambda_{6}\right)+4 \lambda_{2} \lambda_{5}\left(7 \lambda_{3}+6 \lambda_{4}+9 \lambda_{6}\right) \\
&\left.\quad-3 \lambda_{3}^{2}\left(\lambda_{4}+16 \lambda_{6}\right)+\lambda_{3}\left(-4 \lambda_{4}^{2}+4 \lambda_{5}^{2}-54 \lambda_{4} \lambda_{6}+80 \lambda_{6}^{2}\right)\right), \\
& b_{32}=-\left(\lambda_{3}-\lambda_{6}\right)\left(\lambda_{3}+\lambda_{4}+7 \lambda_{6}\right), \\
& b_{33}=8 .
\end{aligned}
$$

Since $\left|\lambda_{j}\right| \leq 1$ for $\lambda \in \Lambda$ and $j \geq 2$,

$$
\left|b_{21}\right| \leq 124, \quad\left|b_{31}\right| \leq 129.5, \quad\left|b_{32}\right| \leq 18 .
$$

It now follows easily from these estimates and inequalities (61) that $\left|f_{j}\right| \leq 10^{4} \sigma^{\prime}$ for all generators $f_{j}$. We have substantially weakened several estimates but, for what follows, this is not essential. 
Thus, if a vector field is $\sigma^{\prime}$-close to centers in complex normal form, then in Kapteyn's normal form the Dulac generators are also small, together with $\sigma^{\prime}$. Let us deduce from this that the vector field $\left(2_{\lambda}\right)$ is $\sigma$-close to centers. First, we factor the Dulac generators $f_{i}$ :

$$
f_{1}=\lambda_{1}, \quad f_{2}=\lambda_{5} p_{6}, \quad f_{3}=\lambda_{2} \lambda_{4} p_{6} p_{4}, \quad f_{4}=\lambda_{2} \lambda_{4} p_{6}^{2} p_{2}^{\prime} p_{2}^{\prime \prime},
$$

where $p_{4}$ and $p_{6}$ are defined in (5), and

$$
p_{2}=\left(\sqrt{\lambda_{6}\left(\lambda_{3}-2 \lambda_{6}\right)}-\lambda_{2}\right)\left(\sqrt{\lambda_{6}\left(\lambda_{3}-2 \lambda_{6}\right)}+\lambda_{2}\right):=\left(\sqrt{q(\lambda)}-\lambda_{2}\right)\left(\sqrt{q(\lambda)}+\lambda_{2}\right):=p_{2}^{\prime} p_{2}^{\prime \prime},
$$

where

$$
q(\lambda)=\lambda_{6}\left(\lambda_{3}-2 \lambda_{6}\right)
$$

The polynomial $f_{4}$ has the largest number of factors, six, among the $f_{i}$. Therefore, each of the $f_{i}$ has at least one factor not exceeding

$$
\sqrt[6]{\max _{i}\left|f_{i}\right|}<5 \sqrt[6]{\sigma^{\prime}}:=\tilde{\sigma} \leq 1
$$

Finally, we show that $\lambda=\left(\lambda_{1}, \ldots, \lambda_{6}\right) \in \Lambda$ is $\sigma$-close to $\Sigma$. For that, we change the coordinates of $\lambda$ so that the new point would be in $\Sigma$. Then we estimate the distance between the new point and $\lambda$, which is going to be small. All or some factors among $\left\{\lambda_{1}, \lambda_{2}, \lambda_{4}, \lambda_{5}, p_{4}, p_{6}, p_{2}^{\prime}, p_{2}^{\prime \prime}\right\}$ whose moduli do not exceed $\tilde{\sigma}$ can be made, by changing the coordinates of $\lambda$, equal to zero. We shall do it in such a way as to make all $f_{i}$ zero. The coordinates of $\lambda$ change in two steps. First, we obtain a point $\lambda^{\prime}$, then $-\lambda^{\prime \prime}$.

1) It is clear that $\left|\lambda_{1}\right|<\tilde{\sigma}$. Set $\lambda_{1}^{\prime}=0 ; \lambda_{6}^{\prime}=\lambda_{6}$; if $\left|\lambda_{5}\right|<\tilde{\sigma}$, then $\lambda_{5}^{\prime}=0$, else $\lambda_{5}^{\prime}=\lambda_{5}$; if $\left|\lambda_{2}\right|<\tilde{\sigma}$, then $\lambda_{2}^{\prime}=0$, else $\lambda_{2}^{\prime}=\lambda_{2}$; if $\left|\lambda_{4}\right|<\tilde{\sigma}$, then $\lambda_{4}^{\prime}=0$, else $\lambda_{4}^{\prime}=\lambda_{4}$; if $\left|p_{6}(\lambda)\right|<\tilde{\sigma}$, then $\lambda_{3}^{\prime}=\lambda_{6}$, else $\lambda_{3}^{\prime}=\lambda_{3}$. Consider the point $\lambda^{\prime}=\left(\lambda_{1}^{\prime}, \ldots, \lambda_{6}^{\prime}\right)$. By definition, $\left|\lambda_{i}^{\prime}-\lambda_{i}\right|<\tilde{\sigma}$, but some polynomials $f_{i}$ may not vanish at $\lambda^{\prime}$. We need to change the point again, but before doing that let us estimate the change in $\left|p_{4}\right|,\left|p_{2}^{\prime}\right|$, and $\left|p_{2}^{\prime \prime}\right|$ under the transition from $\lambda$ to $\lambda^{\prime}$. It is clear that if $\left|p_{4}(\lambda)\right|<\tilde{\sigma}$, then $\left|p_{4}\left(\lambda^{\prime}\right)\right|<7 \tilde{\sigma}$. It is also easy to see that $\left|q\left(\lambda^{\prime}\right)-q(\lambda)\right|<\tilde{\sigma}$. If $\left|p_{2}^{\prime}(\lambda)\right|<\tilde{\sigma}$, then

$$
\left|p_{2}^{\prime}\left(\lambda^{\prime}\right)\right| \leq\left|p_{2}^{\prime}(\lambda)\right|+\left|\lambda_{2}-\lambda_{2}^{\prime}\right|+\left|\sqrt{q\left(\lambda^{\prime}\right)}-\sqrt{q(\lambda)}\right|<2 \tilde{\sigma}+\left|\sqrt{q\left(\lambda^{\prime}\right)}-\sqrt{q(\lambda)}\right| .
$$

We now have two cases:

- If $|q(\lambda)|<\tilde{\sigma}$, then $\left|q\left(\lambda^{\prime}\right)\right|<2 \tilde{\sigma}$. Moreover $\left|\sqrt{q\left(\lambda^{\prime}\right)}-\sqrt{q(\lambda)}\right|<3 \sqrt{\tilde{\sigma}}$ and $\left|p_{2}^{\prime}\left(\lambda^{\prime}\right)\right|<2 \tilde{\sigma}+3 \sqrt{\tilde{\sigma}}<5 \sqrt{\tilde{\sigma}}$.

- If $|q(\lambda)| \geq \tilde{\sigma}$, then, by Claim 1 (p.116), $\left|\sqrt{q\left(\lambda^{\prime}\right)}-\sqrt{q(\lambda)}\right| \leq 2 \sqrt{\tilde{\sigma}}$ and $\left|p_{2}^{\prime}\left(\lambda^{\prime}\right)\right|<2 \tilde{\sigma}+2 \sqrt{\tilde{\sigma}}<5 \sqrt{\tilde{\sigma}}$.

For $\left|p_{2}^{\prime \prime}\left(\lambda^{\prime}\right)\right|$ we have the same estimate: if $\left|p_{2}^{\prime \prime}(\lambda)\right|<\tilde{\sigma}$, then $\left|p_{2}^{\prime \prime}\left(\lambda^{\prime}\right)\right|<5 \sqrt{\tilde{\sigma}}$.

2) Now we set $\lambda_{1}^{\prime \prime}=\lambda_{1}^{\prime}, \lambda_{3}^{\prime \prime}=\lambda_{3}^{\prime}, \lambda_{5}^{\prime \prime}=\lambda_{5}^{\prime}$, and $\lambda_{6}^{\prime \prime}=\lambda_{6}^{\prime}$. If $\lambda_{2}=\lambda_{2}^{\prime}$ and $\lambda_{4}=\lambda_{4}^{\prime}$, then we modify $\lambda_{2}^{\prime}$ and $\lambda_{4}^{\prime}$ in the following manner:

- If $\lambda_{4}^{\prime}=0$ or $\left|p_{4}(\lambda)\right| \geq \tilde{\sigma}$, we set $\lambda_{4}^{\prime \prime}=\lambda_{4}^{\prime}$. Otherwise, we have $\left|p_{4}\left(\lambda^{\prime}\right)\right|<7 \tilde{\sigma}$, and setting $\lambda_{4}^{\prime \prime}=-5\left(\lambda_{3}^{\prime}-\lambda_{6}^{\prime}\right)$, we obtain $\left|\lambda_{4}^{\prime \prime}-\lambda_{4}^{\prime}\right|<7 \tilde{\sigma}$.

- Similarly, if either $\lambda_{2}^{\prime}=0$ or we have, at the same time, $\left|p_{2}^{\prime}(\lambda)\right| \geq \tilde{\sigma}$ and $\left|p_{2}^{\prime \prime}(\lambda)\right| \geq \tilde{\sigma}$, then set $\lambda_{2}^{\prime \prime}=\lambda_{2}^{\prime}$. Otherwise, choose one of the factors $p_{2}^{\prime}(\lambda)$ and $p_{2}^{\prime \prime}(\lambda)$ with smallest modulus. Without loss of generality, we may assume that it is $p_{2}^{\prime}(\lambda)$, i.e., $\left|p_{2}^{\prime}(\lambda)\right|<\tilde{\sigma}$. Then we have $\left|p_{2}^{\prime}\left(\lambda^{\prime}\right)\right|<5 \sqrt{\tilde{\sigma}}$, and setting $\lambda_{2}^{\prime \prime}=\sqrt{q\left(\lambda^{\prime}\right)}$, we obtain $\left|\lambda_{2}^{\prime \prime}-\lambda_{2}^{\prime}\right|<5 \sqrt{\tilde{\sigma}}$.

Now set $\lambda^{\prime \prime}=\left(\lambda_{1}^{\prime \prime}, \ldots, \lambda_{6}^{\prime \prime}\right)$. Each polynomial $f_{i}(\lambda)$ has a factor whose modulus does not exceed $\tilde{\sigma}$. Moreover, $p_{4}, p_{2}^{\prime}$, and $p_{2}^{\prime \prime}$ are factors of only $f_{3}$ and $f_{4}$, which also have factors $\lambda_{2}$ and $\lambda_{4}$. It is now easy to see that all $f_{i}\left(\lambda^{\prime \prime}\right)$ are zero, and that $\lambda_{1}^{\prime \prime}, \lambda_{2}^{\prime \prime}, \lambda_{3}^{\prime \prime}$, $\lambda_{4}^{\prime \prime}, \lambda_{5}^{\prime \prime}, \lambda_{6}^{\prime \prime}$ differ from $\lambda_{1}, \lambda_{2}, \lambda_{3}, \lambda_{4}, \lambda_{5}, \lambda_{6}$ by no more than $\tilde{\sigma}, 5 \sqrt{\tilde{\sigma}}, \tilde{\sigma}, 7 \tilde{\sigma}, \tilde{\sigma}$, and 0 , respectively. Therefore $\left|\lambda-\lambda^{\prime \prime}\right|<15 \sqrt{\tilde{\sigma}}$. 
Since $\bar{\lambda}=\left(\lambda_{2}, \ldots, \lambda_{6}\right) \in \mathbb{S}^{4}$, the distance from $\overline{\lambda^{\prime \prime}}=\left(\lambda_{2}^{\prime \prime}, \ldots, \lambda_{6}^{\prime \prime}\right)$ to $\mathbb{S}^{4}$ is at most $15 \sqrt{\tilde{\sigma}}$, and therefore, using a translation of no more than $15 \sqrt{\tilde{\sigma}}$ along the ray connecting the origin with the point $\left(0, \overline{\lambda^{\prime \prime}}\right) \in \mathbb{R}^{6}$ we can move $\overline{\lambda^{\prime \prime}}$ to $\mathbb{S}^{4}$. It is easy to see that the polynomials $f_{i}$ would still be zero, and the new point would belong to $\Sigma$. Thus, the distance from $\lambda$ to $\Sigma$ is at most $2 \cdot 15 \sqrt{\tilde{\sigma}}<68 \sqrt[12]{\sigma^{\prime}}:=\sigma$. This proves the proposition.

8.2. The Ilyashenko-Llibre theorem for Kapteyn's normal form. Now that we have the relations between the complex normal form and Kapteyn's normal form, we can reformulate Theorem 5 for the vector field $\left(2_{\lambda}\right)$ in Kapteyn's normal form:

Theorem 6. Suppose $0<\delta<1,0<\sigma<1$, and $0<\kappa<1$. If $\lambda \in \Lambda$ and the vector field (2ג) is $\sigma$-distant from centers and $\kappa$-distant from singular fields (in the sense of Definitions 3 and 4), then the number $\delta$-good limit cycles of that vector field is at most

$$
(|\ln \sigma|+1) \exp \left[\exp \left(10^{76} \delta^{-33} \kappa^{-2}\right)\right] .
$$

Proof. Transform the vector field $\left(2_{\lambda}\right)$ in Kapteyn's normal form into complex normal form. Proposition 9 shows that $\delta$-good limit cycles of $\left(2_{\lambda}\right)$ are $\frac{\delta}{36}$-good in complex normal form. Moreover, if $\delta<1$, then $\frac{\delta}{36}<0.1$. If such cycles exist, then, by Proposition 10 , the vector field in complex normal form is at a distance of $\frac{\delta \kappa}{20}<0.1$ from singular fields. Finally, Proposition 11 implies that the vector field in complex normal form is at a distance of $\left(\frac{\sigma}{68}\right)^{12}$ from centers. Now Theorem 5 provides an estimate on the number of $\delta$-good limit cycles of the vector field $\left(2_{\lambda}\right)$ :

$$
12|\ln (\sigma / 68)| \exp \left[\exp \left(0^{25} \cdot 36^{31} \delta^{-31} \kappa^{-2} \delta^{-2} \cdot 20^{2}\right)\right] \text {. }
$$

It is easy to see that estimate (64) is rougher.

8.3. Proof of Proposition 2. As we mentioned in Section 5, a result similar to Proposition 2 is proved in [5. Below, we shall state it and also prove Proposition 2 ,

Consider equation (49) in complex normal form and switch to polar coordinates $(R, \phi)$, $z=\operatorname{Re}^{i \phi}([\mathbf{5}])$. We then have a system of equations:

$$
\dot{R}=R\left(\lambda_{1}+F(A, B, C, \phi) R\right), \quad \dot{\phi}=1+G(A, B, C, \phi) R,
$$

where

$$
F(A, B, C, \phi)=\operatorname{Re} h(A, B, C, \phi), \quad G(A, B, C, \phi)=\operatorname{Im} h(A, B, C, \phi)
$$

and

$$
h(A, B, C, \phi)=A e^{i \phi}+B e^{-i \phi}+C e^{-3 i \phi} .
$$

The isocline $\{\dot{\phi}=0\}$ is then given by the equation

$$
R=-\frac{1}{G(A, B, C, \phi)} .
$$

Set $\beta^{\prime}=\frac{\delta^{14} \kappa}{10^{10}}$ and consider the strip

$$
\Pi_{\beta^{\prime}}=\left\{(\phi, R) \mid R \in\left[-\frac{1}{G(A, B, C, \phi)}-\beta^{\prime} ;-\frac{1}{G(A, B, C, \phi)}\right]\right\} .
$$

In [5], one finds the following results:

Proposition 12. All limit cycles of the vector field (49) encircling the origin are contained in the domain $\{\dot{\phi}>0\}$.

Proposition 13 ([5, Lemma 12]). Suppose $\{\delta, \kappa\} \subset(0,0.1)$. If the vector field (49) in complex normal form is $\kappa$-distant from singular fields in the sense of Definition 14, then its $\delta$-good limit cycles do not intersect the strip $\Pi_{\beta^{\prime}}$. 
We shall show that these results imply Proposition 2 Indeed, switch from $\left(2_{\tilde{\lambda}}\right)$ to complex normal form. The vector field (49) in complex normal form is at a distance of $\frac{\delta \kappa}{20}<0.1$ from singular fields. Any $\delta$-good limit cycle of the vector field $(2 \tilde{\lambda})$ is $\frac{\delta}{36}$-good in complex normal form. By Propositions 12 and 13, such a cycle is contained in the domain

$$
\begin{aligned}
\tilde{\Omega}_{\beta^{\prime}}= & \left\{(\phi, R) \mid 0<R<-\frac{1}{G(A, B, C, \theta)}-\beta^{\prime} \text { if } G(A, B, C, \phi)<0\right\} \\
& \cup\{(\phi, R) \mid 0<R<+\infty \text { if } G(A, B, C, \phi) \geq 0\},
\end{aligned}
$$

where

$$
\beta^{\prime}=\frac{\left(\frac{\delta}{36}\right)^{14} \frac{\delta \kappa}{20}}{10^{10}}<\frac{3 \delta^{15} \kappa}{10^{34}}=3 \beta .
$$

Switching to complex normal form does not increase distances by more than 3 times (Proposition 8), and the isocline $\{\dot{\theta}=0\}=\left\{r=-1 / g_{\tilde{\lambda}}(\theta)\right\}$ for system $\left(6_{\tilde{\lambda}}\right)$ transforms into the isocline $\{\dot{\phi}=0\}=\{R=-1 / G(A, B, C, \phi)\}$ for system (65). Therefore, by (66), if a $\delta$-good limit cycle of the vector field $(2 \tilde{\lambda})$ is not contained in $\Omega_{\beta}$, then, after switching to complex normal form, its image is not contained in $\tilde{\Omega}_{\beta^{\prime}}$. The obtained contradiction proves Proposition 2

8.4. Proof of Theorem 2, To prove Theorem 2, we apply Theorem 1 assuming that $\sigma<\exp \left(-10^{73} \kappa^{-2} \delta^{-33}\right):=\sigma_{0}$, together with Theorem 6 for $\sigma_{0} \leq \sigma<1$. It is not difficult to check that estimate (64) in the latter case majorizes estimate (9) in the former. We also have

$$
\left(\left|\ln \sigma_{0}\right|+1\right) \exp \left[\exp \left(10^{76} \delta^{-33} \kappa^{-2}\right)\right] \leq \exp \left[\exp \left(10^{77} \delta^{-33} \kappa^{-2}\right)\right]
$$

which proves Theorem 2

\section{APPENDix (COMPUTER-Aided CALCUlations)}

In this paper, several calculations were done using the computer algebra system Mathematica ${ }^{\circledR}$. Below, we shall describe the corresponding code.

Decomposition of canonical generators by the Dulac generators. We shall describe the computer program used to calculate the entries $a_{i j}$ of the matrix $A$ (see (22)). As was shown in Subsection [6.3, the functions $g_{i}(\lambda)$ are the values $v_{i}(\lambda, 2 \pi)$ of the solutions $v_{i}(\lambda, \theta)$ of equations (35) with initial conditions (36), and those equations are solved recurrently. The entries $a_{i j}$ of $A$ are the coefficients in the decomposition of the functions $g_{i}(\lambda)$ by the Dulac generators $f_{1}, \ldots, f_{4}$, which are given by (4) and (5). The Mathematica program performing these calculations is described below. In it, we replaced $\theta$ by $\mathrm{x}$ and $\lambda_{i}$ by ti.

Definition of functions $f_{\lambda}(\theta), g_{\lambda}(\theta), R_{i}(\lambda, \theta)$.

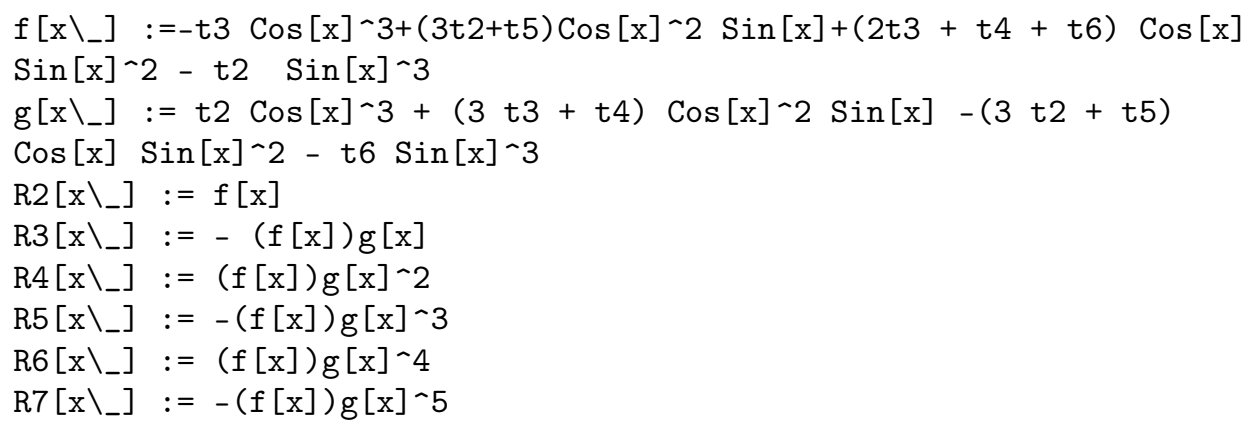


Solution of differential equations for $v_{i}(\lambda, \theta)$.

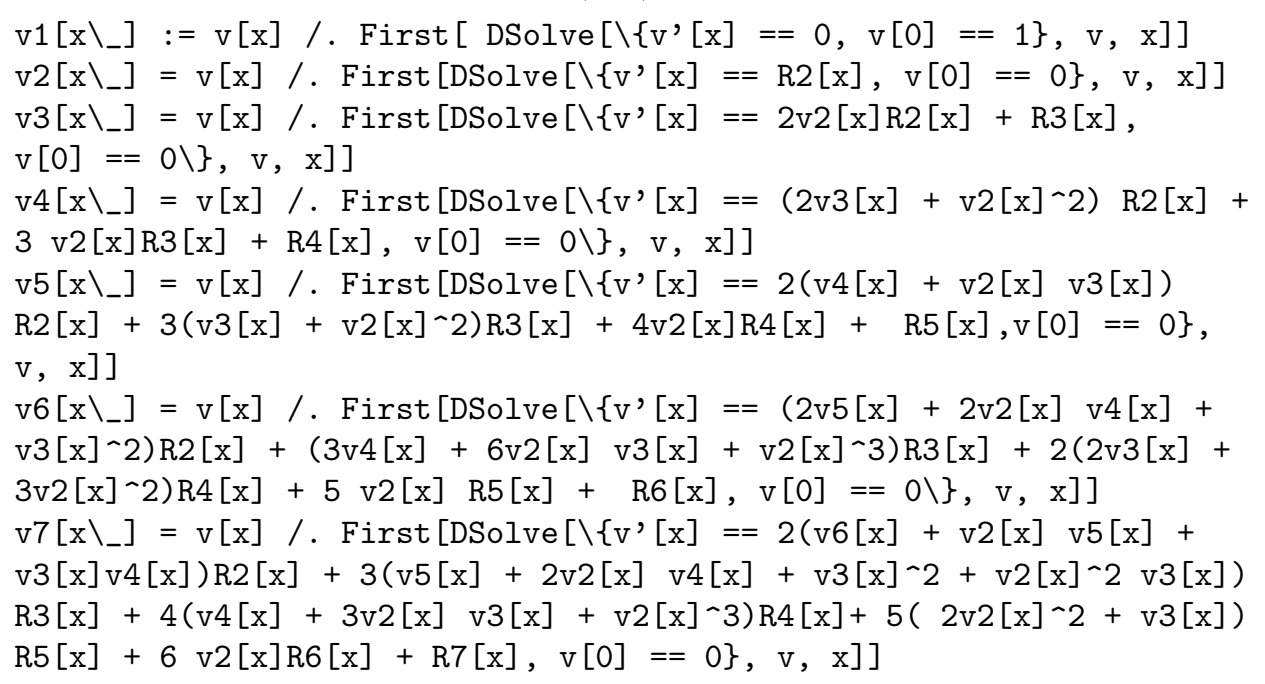

Determination of generators $f_{2}, f_{3}, f_{4}$ of the Bautin ideal for $\lambda_{1}=0$.

$\mathrm{f} 2:=\mathrm{t} 5(\mathrm{t} 3-\mathrm{t} 6)$

$f 3:=t 2 t 4(t 3-t 6)(t 4+5(t 3-t 6))$

$f 4:=t 2$ t4 (t3 - t6) $2(t 6(t 3-2 t 6)-t 2 \sim 2)$

Substitution $\theta=2 \pi$ for computing the Poincaré map. Now vi $[\mathrm{x}]$ coincides with $g_{i}$.

$\mathrm{x}=2 \mathrm{Pi}$

A bypass for determination of $a_{44}$ ("computer guessing"). We substitute the values $\lambda_{5}=0$ and $\lambda_{4}=-5\left(\lambda_{3}-\lambda_{6}\right)$ in $g_{7}$ and $f_{4}$. This makes the generators $f_{2}$ and $f_{3}$ vanish, so that $a_{44}$ can be found by dividing $g_{7}$ by $f_{4}$.

$\mathrm{a} 44=$ First $[$ First $[$ PolynomialReduce $[\mathrm{v} 7[\mathrm{x}] / . \mathrm{t5}->0 /$. t4 $->-5(\mathrm{t} 3-$

$\mathrm{t} 6),\{\mathrm{f} 4 / . \mathrm{t} 5 \rightarrow 0 / . \mathrm{t} 4->-5(\mathrm{t} 3-\mathrm{t} 6)\},\{\mathrm{t} 2, \mathrm{t} 3, \mathrm{t} 6\}]]]$

Decomposition of $g_{3}, g_{5}, g_{7}$ by $f_{2}, f_{3}, f_{4}$. For $g_{7}$ we use the already determined coefficient $a_{44}$ of $f_{4}$.

PolynomialReduce $[v 3[x],\{f 2, f 3, f 4\},\{t 2, t 3, t 4, t 5, t 6\}]$

PolynomialReduce $[v 5[x],\{f 2, f 3, f 4\},\{t 2, t 3, t 4, t 5, t 6\}]$

PolynomialReduce[v7[x] - a44 f4, $\{\mathrm{f} 2, \mathrm{f} 3, \mathrm{f} 4\},\{\mathrm{t} 2, \mathrm{t} 3, \mathrm{t} 4$, t5, t6 6 ]

The output of the last 4 lines of this program gives decompositions of $g_{3}, g_{5}$ and $g_{7}-a_{44} f_{4}$ by the generators $\left\{f_{i}\right\}$ with coefficients $a_{i j}(\lambda)$, given by (39), with zero remainders. It turned out that Mathematica's command PolynomialReduce does not decompose $g_{7}$ by the $f_{i}$ without remainder. For that reason, to find $a_{44}$ we used "guessing" (see above), and successfully decomposed $g_{7}-a_{44} f_{4}$ by the $\left\{f_{i}\right\}$, without remainder, using PolynomialReduce.

Matrix of transition from Żołądek generators to Dulac generators. Now we describe the program for calculating the entries $b_{i j}$ of the transition matrix $B$, defined by (62). In that program, we modified the notation as follows: A, B, K stand for $A_{0}, B_{0}$, and $C_{0}$, respectively (see (56) $) ; \mathrm{h} 2, \mathrm{~h} 3, \mathrm{~h} 4$ denote $h_{2}^{0}, h_{3}^{0}$ and $h_{4}^{0}$ (see (60)); and $\mathrm{f} 2, \mathrm{f3}, \mathrm{f} 4$ are the Dulac generators $f_{2}, f_{3}$ and $f_{4}$ (see (4) and (5)). The letter I 
stands for the imaginary unit. Here is the program's code:

Define $A_{0}, B_{0}, C_{0}, h_{i}^{0}, f_{i}$.

$\mathrm{A}:=(\mathrm{t} 3+\mathrm{t} 4-\mathrm{I} \mathrm{t5}-\mathrm{t} 6) / 4$

$B:=(-2 t 3+2 t 6) / 4$

$\mathrm{K}:=(4 \mathrm{It} 2-3 \mathrm{t} 3-\mathrm{t} 4+\mathrm{It} 5-\mathrm{t} 6) / 4$

$\mathrm{h} 2:=$ ComplexExpand $[\operatorname{Im}[\mathrm{A} * \mathrm{~B}]]$

h3 $:=$ FullSimplify $[$ ComplexExpand $[\operatorname{Im}[(2 A+$ Conjugate $[B])$

$(\mathrm{A}-2$ Conjugate $[\mathrm{B}])($ Conjugate $[\mathrm{B}] * \mathrm{~K})]]]$

h4 := FullSimplify [ComplexExpand $[\operatorname{Im}[(2 \mathrm{~A}+$ Conjugate $[B])$

$(\mathrm{B} *$ Conjugate $[\mathrm{B}]-\mathrm{K} *$ Conjugate $[\mathrm{K}]) *$ Conjugate $[\mathrm{B}] \backslash \sim 2 * \mathrm{~K}]]]$

f2 $:=\mathrm{t} 5 *(\mathrm{t} 3-\mathrm{t} 6)$

f3 $:=$ t2 $* t 4 *(t 3-t 6)(t 4+5(t 3-t 6))$

$\mathrm{f} 4:=\mathrm{t} 2 * \mathrm{t} 4 *(\mathrm{t} 3-\mathrm{t} 6) \sim 2(\mathrm{t} 6 *(\mathrm{t} 3-2 \mathrm{t} 6)-\mathrm{t} 2 \sim 2)$

Decompose $h_{i}^{0}$ by the $\left\{f_{i}\right\}$. To decompose $h_{4}^{0}$ we again use "guessing" as in the decomposition of the canonical generators by the Dulac generators.

String1 := First [PolynomialReduce $[\mathrm{h} 2,\{\mathrm{f} 2, \mathrm{f} 3, \mathrm{f} 4\}$, $\{t 2, t 3, t 4, t 5, t 6\}]]$

String2 := First [PolynomialReduce $[\mathrm{h} 3,\{\mathrm{f} 2, \mathrm{f} 3, \mathrm{f} 4\}$,

$\{\mathrm{t} 2, \mathrm{t} 3, \mathrm{t} 4, \mathrm{t} 5, \mathrm{t} 6\}]]$

$\mathrm{a}:=$ First $[$ First $[$ PolynomialReduce $[\mathrm{h} 4 /$. t5 $->0 /$.

$\mathrm{t} 4->-5(\mathrm{t} 3-\mathrm{t} 6)$,

$\{\mathrm{f} 4 / . \mathrm{t} 5 \rightarrow 0 / . \mathrm{t} 4 \rightarrow-5(\mathrm{t} 3-\mathrm{t} 6)\},\{\mathrm{t} 2, \mathrm{t} 3, \mathrm{t} 6\}]]]$

String3prelim $:=$ First [PolynomialReduce[h4 - a f4,

$\{f 2, f 3, f 4\},\{t 2, t 3, t 4, t 5, t 6\}]]$

String3 := \{String3prelim[[1]], String3prelim[[2]], a\}

Put the coefficients obtained in the matrix $B^{-1}$ and invert it.

BInverse := \{String1, String2, String3\}

$B=$ Simplify [Inverse [BInverse $]]$

The output of this program is the matrix with entries (63).

\section{ACKNOWLEDGMENTS}

The author is deeply grateful to Yu. S. Ilyashenko for posing the problem, his attention during the work on it, and fruitful discussions, as well as for providing an ideal creative environment for writing this paper.

\section{REFERENCES}

[1] W. A. Coppel, A survey of quadratic systems, J. Differential Equations 2 (1966), 293-304. MR0196182 (33:4374)

[2] H. Dulac, Détermination et intégration d'une certaine classe d'équations différentielles ayant pour point singulier un centre, Bull. Soc. Math. France (2) 32 (1908), 230-252.

[3] A. Fishkin, On the number of zeros of an analytic perturbation of the identically zero function on a compactum, Mat. Zametki 85 (2009), no. 1, 110-118; English transl., Math. Notes 85 (2009), no. 1-2, 101-108. MR 2547968

[4] M. Hervé, Several complex variables. Local theory. Oxford University Press, Bombay, 1963. $\operatorname{MR} 0151632(27: 1616)$

[5] Yu. Ilyashenko and J. Llibre, A restricted version of Hilbert's 16th problem for quadratic vector fields, arXiv:0910.3443v1 [math.DS].

[6] Yu. Ilyashenko and S. Yakovenko, Counting real zeros of analytic functions satisfying linear differential equations, J. Differential Equations 126 (1996), 87-105. MR1382058 (97a:34010) 
[7] Yu. Ilyashenko and S. Yakovenko, Lectures on Analytic Differential Equations, Graduate Studies in Mathematics 86, Amer. Math. Soc., Providence, RI, 2008. MR2363178 (2009b:34001)

[8] W. Kapteyn, On the midpoints of integral curves of differential equations of the first degree, Nederl. Akad. Wetensch. Verslag. Afd. Natuurk. Konikl. Nederland 19 (1911), 1446-1457. (Dutch)

[9] W. Kapteyn, New investigations on the midpoints of integrals of differential equations of the first degree, Nederl. Akad. Wetensch. Verslag. Afd. Natuurk 20 (1912), 1354-1365; 21, 27-33. (Dutch)

[10] R. Roussarie, Bifurcation of planar vector fields and Hilbert's sixteenth problem, Birkhäuser, Basel, 1998. MR 1628014 (99k:58129)

[11] Pingguang Zhang, Quadratic systems with two foci, Appl. Math. J. Chinese Univ. 14A (1999), 247-253. (Chinese) MR.1716432 (2000f:34059)

[12] Pingguang Zhang, On the distribution and number of limit cycles for quadratic systems with two foci, Acta Math. Sinica (Chin. Ser.) 44 (2001), 37-44. (Chinese) MR.1819975 (2001m:34073)

[13] Pingguang Zhang, On the distribution and number of limit cycles for quadratic systems with two foci, Qual. Theory Dyn. Syst. 3 (2002), 437-463. MR.2020695 (2004i:34071)

[14] H. Żołądek, Quadratic systems with center and their perturbations, J. Differential Equations 109 (1994), no. 2, 223-273. MR.1273302 (95b:34047)

M. V. Lomonosov Moscow State University

E-mail address: fishkinalexey@gmail.com 